HU-P-D149

\title{
Quantifying Time Awake Posturographically
}

\section{Pia Forsman}

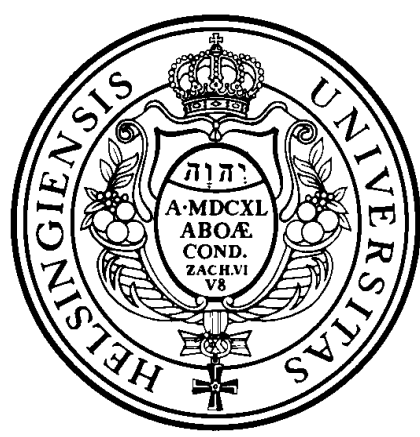

Electronics Research Unit Department of Physics Faculty of Science University of Helsinki Helsinki, Finland

for public criticism in Auditorium D101 of the Department of Physics (Physicum), on September 12th, 2008, at 12 o'clock noon. 
Supervisor:

Professor Edward Hæggström, PhD, MBA

Electronics Research Unit, Department of Physics, University of Helsinki, Finland

Co-supervisors:

Docent Esko Toppila, PhD

Department of Work Environment Development, Finnish Institute of Occupational Health, Helsinki, Finland

Professor Ilmari Pyykkö, MD

Department of Otolaryngology, University of Tampere, Finland

Reviewers:

Professor Kiti Müller, MD

The Brain and Work Research Centre, Finnish Institute of Occupational Health, Helsinki, Finland

Docent Pertti Era, PhD

Department of Health Sciences, University of Jyväskylä, Finland

Opponent:

Professor Hannes Petersen, MD

Department of Otorhinolaryngology H \& N Surgery, Landspítali University Hospital, Iceland

ISBN 978-952-10-3924-9 (printed version)

ISSN 0356-0961

Helsinki 2008

Helsinki University Printing House (Yliopistopaino)

ISBN 978-952-10-3925-6 (PDF version)

http://ethesis.helsinki.fi/

Helsinki 2008

Electronic Publications @ University of Helsinki (Helsingin yliopiston verkkojulkaisut) 
P Forsman: Quantifying Time Awake Posturographically, University of Helsinki, 2008, 43 p.+appendices. University of Helsinki Report Series in Physics, HU-P-D149, ISSN 0356-0961, ISBN 978-952-10-3924-9, (printed version), ISBN 978-952-10-3925-6 (PDF version)

Classification (INSPEC): E0240H, A8730, B8520, A8745D

Keywords (INSPEC): Accident prevention, sleep loss, quantitative sleepiness testing, traffic safety, occupational safety, public safety, balance testing, quantitative posturography 


\section{ABSTRACT}

This thesis focuses on the issue of testing sleepiness quantitatively. The issue is relevant to policymakers concerned with traffic- and occupational safety; such testing provides a tool for safety legislation and -surveillance. The findings of this thesis provide guidelines for a posturographic sleepiness tester.

Sleepiness ensuing from staying awake merely $17 \mathrm{~h}$ impairs our performance as much as the legally proscribed blood alcohol concentration $0.5 \%$ does. Hence, sleepiness is a major risk factor in transportation and occupational accidents. The lack of convenient, commercial sleepiness tests precludes testing impending sleepiness levels -contrary to simply breath testing for alcohol intoxication. Posturography is a potential sleepiness test, since clinical diurnal balance testing suggests the hypothesis that time awake could be posturographically estimable. Relying on this hypothesis this thesis examines posturographic sleepiness testing for instrumentation purposes.

Empirical results from 63 subjects -for whom we tested balance with a force platform during wakefulness for maximum $36 \mathrm{~h}$ - show that sustained wakefulness impairs balance. The results show that time awake is posturographically estimable with $88 \%$ accuracy and 97\% precision -which validates our hypothesis. Results also show that balance scores tested at 13:30 hours serve as a threshold to detect excessive sleepiness. Analytical results show that the test length has a marked effect on estimation accuracy: $18 \mathrm{~s}$ tests suffice to identify sleepiness related balance changes, but trades off some of the accuracy achieved with 30 s tests.

The procedure to estimate time awake relies on equating the subject's test score to a reference table (comprising balance scores tested during sustained wakefulness, regressed against time awake). Empirical results showed that sustained wakefulness explains $60 \%$ of the diurnal balance variations, whereas the time of day explains $40 \%$ of the balance variations. The latter fact implies that time awake estimations also must rely on knowing the local times of both test and reference scores.

Hypothesis: Sustained wakefulness impairs balance.

Thesis: Posturographic balance tests estimate time awake.

Value statement: This thesis creates value for policymakers concerned with traffic- and occupational safety by providing guidelines for a posturographic sleepiness tester. 


\section{ACKNOWLEDGEMENTS}

First, my sincerest thanks to all volunteers in this study -thank you for a most important contribution to this work!

Special thanks to Prof. Edward Hæggström, my supervisor, for his expert guidance, and for always lending me his support and expertise in applied physics. I also thank Doc. Esko Toppila and Prof. Ilmari Pyykkö, my co-supervisors, for putting their expertise on balance at my disposal.

Thanks to my co-writers for their constructive comments on these multidisciplinary issues; especially to MSc. Anders Wallin, for assisting in instrumentation- and analysis issues, and to MSc. Aino Tietäväinen for performing part of the measurements.

I also wish to thank Prof. Kiti Müller and Doc. Pertti Era, reviewers of the thesis, for their constructive comments.

This thesis was realized during my tenure at the Finnish Institute of Occupational Health. The institute has always looked very positively on my research, which is gratefully acknowledged.

Part of this study was realized at the Electronics Research Unit, Department of Physics, University of Helsinki. I thank Prof. Juhani Keinonen, head of the department, and Prof. Heimo Saarikko, head of the unit, for allowing the facilities at my disposal. Part of this study was realized at the Department of Physics, Finnish Institute of Occupational Health. I thank the department for putting the posturographic equipment at my disposal, and for enabling the research.

Finally, thanks to my husband Anders, for always standing by with encouragements.

Helsinki, July 2008

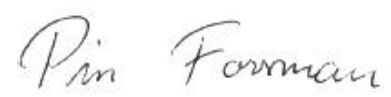

Pia Forsman, Lic.Phil. 


\section{ABBREVIATIONS}

$\begin{array}{ll}\text { ANOVA } & \text { Analysis of variance } \\ A P & \text { Anteroposterior direction } \\ B A C & \text { Blood alcohol concentration } \\ F D & \text { Fractal dimension of sway } \\ C F F & \text { Critical flicker fusion } \\ C N S & \text { Central nervous system } \\ C o M & \text { Center of mass } \\ C o P & \text { Center of pressure } \\ C V & \text { Coefficient of variation } \\ \triangle T & \text { Time awake estimation accuracy } \\ E E G & \text { Electroencephalogram } \\ E S S & \text { Epworth sleepiness scale } \\ K S S & \text { Karolinska sleepiness scale } \\ L E D & \text { Light emitting diode } \\ M L & \text { Lateral direction } \\ M C A & \text { Most common sway amplitude } \\ N P V & \text { Negative predictive value } \\ M S L T & \text { Multiple sleep latency test } \\ M W T & \text { Maintenance of wakefulness test } \\ P I D & \text { Proportional-, integral-, and derivative } \\ P P V & \text { Positive predictive value } \\ P V T & \text { Psychomotor vigilance task } \\ S D & \text { Standard deviation } \\ S L I P M & \text { Single-link inverted pendulum } \\ S N R & \text { Signal-to-noise ratio } \\ T O C & \text { Time interval for open loop stance control }\end{array}$




\section{CONTENTS}

ABSTRACT

ACKNOWLEDGEMENTS

ABBREVIATIONS iii

ORIGINAL PUBLICATIONS 1

AUTHOR'S CONTRIBUTION 1

PUBLICATION SUMMARIES 1

1 INTRODUCTION 2

2 SLEEPINESS, BALANCE, AND POSTUROGRAPHY 4

SLEEPINESS

2.1 Nature of Sleepiness 4

2.2 Factors Altering Sleepiness 5

2.3 Classifying and Measuring Sleepiness 5

BALANCE $\quad 6$

$\begin{array}{ll}2.4 \text { Nature of Balance } & 6\end{array}$

2.4.1 Musculoskeletal System $\quad 7$

2.4.2 Vestibular, Proprioceptive, and Visual Systems 8

2.4.3 CNS and Balance Control $\quad 8$

2.5 Factors Altering Balance $\quad 9$

2.5.1 Sleepiness, Circadian Rhythm, Stress, and Fatigue 9

2.5.2 Intrinsic and Extrinsic Factors 9

$\begin{array}{ll}\text { POSTUROGRAPHY } & 10\end{array}$

2.6 Force Platform and $C o P$ Trajectories 10

2.7 Analyzing $C o P$ Trajectories 12

$\begin{array}{ll}\text { 2.7.1 Global Parameters } & 12\end{array}$

$\begin{array}{lr}\text { 2.7.2 Structural Parameters } & 12\end{array}$

2.8 Physical Models 13

2.9 Validity and Repeatability of Posturography 16

2.10 Balance Testing During Sustained Wakefulness 16

SYNTHESIZING A POSTUROGRAPHIC SLEEPINESS TESTER 16

3 STUDY AIM 18

4 SUBJECTS AND METHODS 19

$\begin{array}{ll}4.1 \text { Subjects } & 19\end{array}$

4.2 Study Designs 19

4.3 Balance Testing and Platform Functioning 20

4.4 CFF Testing 21

4.5 Quantifying Monotone Balance Impairments (Study I) 22

4.6 Estimating Time Awake (Study II) 23

4.7 Quantifying Repeatability and Daytime Changes (Study III) 23

4.8 Validating Posturographic Sleepiness Testing (Study IV) 23

4.9 Quantifying Test Accuracy Versus Test Length (Study V) 24

4.10 Improving Test Validity (Study VI) 24

5 MAIN RESULTS 24

5.1 Monotone Balance Impairments (Study I) 24

5.2 Time Awake Estimates (Study II) 26

5.3 Repeatability and Daytime Changes (Study III) 28

5.4 Validity of Posturographic Sleepiness Testing (Study IV) 29

5.5 Test Accuracy Versus Test Length (Study V) 31

5.6 Improved Test Validity (Study VI) 31

6 DISCUSSION 33 
6.1 Need for Study and Contribution of the Thesis

6.2 Quantifying Time Awake Posturographically

6.3 The Physiologic Link Between Balance and Successful Sleepiness Testing

6.4 Limitations and Implications of the Thesis

6.5 Future Research 


\section{ORIGINAL PUBLICATIONS}

I. E Hæggström, P Forsman, A Wallin, E Toppila and I Pyykkö. Evaluating Sleepiness using Force Platform Posturography. IEEE Trans Biomed Eng 2006;53(8):1578-85.

II. P Forsman, A Wallin, A Tietäväinen and E Hæggström. Posturographic sleepiness monitoring. J Sleep Res 2007;16(3):259-61.

III. P Forsman, E Hæggström, A Wallin, E Toppila, I Pyykkö. Daytime changes in postural stability and repeatability of posturographic measurements. J Occup Environ Med 2007;49(6):591-6.

IV. P Forsman, A Tietäväinen, A Wallin, and E Toppila, E Hæggström. Validating posturographic sleepiness monitoring. Submitted.

V. P Forsman, E Hæggström and A Wallin. Reducing trial length in force platform posturographic sleep deprivation measurements. Meas Sci Technol 2007;18:28937.

VI. P Forsman, A Tietäväinen, A Wallin, E Toppila, and E Hæggström. Balance control impairment during sustained waking allows posturographic sleepiness testing. J Biomechanics. In press.

\section{AUTHOR'S CONTRIBUTION}

The thesis's author designed studies I, III, and IV, performed the measurements in I and III, supervised the measurements in IV, analyzed the results in I-VI, ideated and designed publications II-IV and VI, authored publications II-VI, and co-authored publication I.

\section{PUBLICATION SUMMARIES}

Paper I shows that human balance impairs monotonically with increasing time awake, concurrent with the diurnal balance variation. With 21 subjects we show that the rate of monotone impairment allows separating balance scores recorded at $5 \mathrm{~h}$ intervals with $95 \%$ probability, and suggest that a 30 s posturographic balance test estimates time awake with $5 \mathrm{~h}$ accuracy provided one knows the time of day of the test. This paper is important as it verifies the negative and monotone correlation between balance and time awake. 
Paper II presents a method to estimate time awake with posturographic balance tests. With 20 subjects we show that time awake is estimable with $69 \%$ positive predictive value, $56 \%$ sensitivity, and $96 \%$ specificity. This paper is important as it presents the steps to estimate time awake, and as it evaluates the method.

With 30 subjects paper III shows that the balance scores for the time awake estimates in studies I and II are repeatable across a month, and that the scores are responsive to daytime changes in balance. The paper suggests employing balance scores recorded at 13:30 hours as reference values when testing time awake for occupational safety. This paper is important as it verifies the long run repeatability of posturographic tests, and as it presents a score threshold that signals excessive sleepiness.

Paper IV validates posturographic time awake testing. With a one-month protocol in which we tested 12 subjects' balance once a day during a week, and once a week during a month, we show that posturographic balance tests estimate time awake with $86 \%$ accuracy and $97 \%$ precision. This paper is important as it replicates the earlier studies and their results, and thus validates the method.

Paper V quantifies the impact of the balance test length on the time awake estimate accuracy. We show that if accuracy is traded for test speed, $18 \mathrm{~s}$ tests separate balance scores recorded at $5 \mathrm{~h}$ interval with $95 \%$ probability. This paper is important as it determines the method efficiency in terms of posturographic sensitivity against posturographic test speed.

Paper VI models balance as an inverted pendulum and shows that quantifying the

Central Nervous System controlled body Center of Mass movements improves the sensitivity of the posturographic time awake estimates. This paper is important as it improves the method validity by linking balance control to the physiologic impact of increasing time awake.

\section{INTRODUCTION}

This section reviews the hazards of sleepiness, the magnitude of the issue, the commercially available safety precautions, and the need for convenient sleepiness tests.

Sleepiness is a growing safety issue in the industrialized world. One understands the hazards of sleepiness from an analogy with drunken driving: driving after $17 \mathrm{~h}$ of wakefulness equals driving with $0.5 \%$ o blood alcohol concentration $(B A C)$; driving after 20 $\mathrm{h}$ of wakefulness equals driving with 1.0\%o BAC [1-3] (wakefulness means hours awake basically the hours that have elapsed since the person got out of bed). Most industrialized countries legislate against driving and working with $0.5 \%$ o $B A C$ to prevent accidents. Therefore it would be logical to set a $17 \mathrm{~h}$ waking limit -to prevent sleepiness related accidents [4]. Unfortunately, contrary to breath-testing for the $B A C$, there is no analogous simple sleepiness test [5-7]. 
To find a simple and quantitative sleepiness test is important [6-8], because people tend to underrate the hazards of sleepiness. Adult drivers exemplify this: $60 \%$ of them drive while sleepy; $37 \%$ of them have nodded off or fallen asleep at least once while driving; $13 \%$ of them nod off or fall asleep at least once a month while driving [9]. Shift workers again -about 49 million people covering $15 \%$ of the US and EU workforce [10, 11]- often enter the shift after $16 \mathrm{~h}$ of wakefulness [12], and then drive home after $25 \mathrm{~h}$ of wakefulness. Given the hazards of tiredness and the imprudent attitude towards it, explains why sleepiness causes accidents. Sleepiness causes even $20 \%$ of all traffic accidents [1315], and even $50 \%$ of all occupational accidents [16] -either on roadways, in air, in hospitals, or in the industry. Moreover, the trade-off between sleep and sustained wakefulness has mushroomed for the past decade: on average we sleep $2 \mathrm{~h}$ less than 40 years ago, and $20 \%$ of us feel this is less than needed [9, 17]. Global newspapers highlight the hazards of, and legal actions against, drowsy driving [18, 19]. Finnish newspapers begin to highlight the same issues, indicating that sleepy driving is an issue in Finland as well [20, 21]. The magnitude of the safety issue explains the ongoing research [8], regulatory [22], and legislative [6] efforts to minimize the public health and safety hazards of sleepiness. In the US there is already legislation criminalizing driving while tired [23] but measuring sleepiness with a test that responds quantitatively to sustained wakefulness is still a challenge [5-7].

Sleepiness is the tendency to fall asleep [24], which grows with increasing time awake $[23,25]$. One way to improve safety is to alarm the users, e.g. drivers, when dozing off nears. This technique is available on the market implemented using infrared cameras and sensors that continuously track eyelid movement or head inclination [26]. However, this is not true accident prevention -which is to stop the driving or working user before sleepiness causes dozing [4]. The shortcoming results from the fact that well before dozing, a person's accident proneness rises due to impaired performance [1-3]. A way to solve this dilemma is to test a sleepiness marker that responds quantitatively to increasing time awake. This allows scoring the current sleepiness, as well as predicting if alarming performance impairment (and thus accident proneness) will arise during the drive or work shift. Available tests that score sleepiness (reviewed in 2.3) share some issues. First, most tests rely on scoring sleepiness with questionnaires, or scoring it from task based performance. This means that the reliability is questionable as the test results strongly depend on personal factors -both the subject's compensatory strategies for sleepiness, and the subject's motivation may temporarily override a performance decrement [5]. Second, objective tests are lengthy or cumbersome to take. Wrapping up, the market needs a quantitative, fast, and simple sleepiness test. Force platform posturography -a clinical tool to test balance- provides a candidate because balance impairs due to sleepiness (reviewed in 2.5.1 and 2.10).

This thesis examines quantitative time awake testing with posturography for instrumentation purposes. The rationale is that time awake is a relevant parameter in 
sleepiness, and that balance is a physiologic function, which allows designing a test that 1) does not rely on task based performance, and in which 2) compensation and motivation cause as little bias as possible. Moreover, posturography is well documented, and the test is fast and simple to perform. This thesis focuses on acute sleepiness that sustained wakefulness evokes. Cumulative sleepiness that recurrent sleep disruptions cause is out of scope, even if it is more common in the society. The reason for focusing on acute sleepiness is that it depends on our homeostatic sleep drive, which correlates positively with our time awake. Moreover, acute sleepiness prevails among exposed professions, e.g. shift workers [12], and -importantly- acute sleepiness is also faster and easier to generate for study purposes.

\section{SLEEPINESS, BALANCE, AND POSTUROGRAPHY}

This section reviews the theory of three core concepts of a force platform posturographic sleepiness tester: sleepiness, balance, and posturography. The review omits cumulative sleepiness, weekend effects on sleepiness, dynamic posturography, and physical models (except the single-link inverted pendulum model) since this thesis did not examine or employ these concepts.

\section{SLEEPINESS}

This section reviews sleepiness components, available sleepiness testing, and factors to consider when designing a sleepiness study.

\subsection{Nature of Sleepiness}

Sleepiness is our tendency to fall asleep -our sleep drive [24, 27, 28]. Sleepiness is a normal physiological state that depends on the time of day, because whether we have slept or not, sleepiness peaks at night (2-7 am) and in the afternoon (2-5 pm) [28]. This circadian rhythm exhibits large personal differences: people group as morning-, neither-, or evening types and exhibit different circadian phase; people with a delayed phase exhibit larger circadian amplitude [29]. Separately superimposed on the normal circadian sleepiness are the consequences of prolonged wakefulness (causing acute sleepiness) and recurrent sleep disruptions (causing cumulative sleepiness) [28]. Both factors increase sleepiness gradually with continued wakefulness. Moreover, sleep inertia increases sleepiness immediately upon waking, but generally dissipates in $2 \mathrm{~h}$ [30, 31]. Summing up, acute sleep deprivation, cumulative sleep deprivation, circadian rhythm, sleep homeostasis, and sleep inertia can increase sleepiness. 
As sleepiness increases, the probability of micro sleeps increases [32] because cortical alertness decreases $[33,34]$. Even if sleepiness has not gotten to the state of micro sleeps, decreasing alertness reduces cognitive performance [1-3].

The presence of the circadian rhythm requires that a sleepiness tester measures a sleepiness marker responsive to increasing homeostatic sleep drive [23]. The homeostatic sleep drive is a function of time awake: increasing waking increases the drive [25]. If the tester does not measure this component, the circadian sleepiness may mask and hamper the interpretation of the measurement results. The individual differences in circadian phase and amplitude also mean that a sleepiness tester probably requires individual calibration -the sleepiness marker may not be comparable to population means.

\subsection{Factors Altering Sleepiness}

This section reviews factors that bias test results if not accounted for in the study design.

In a healthy person the sleepiness level mainly depends on the current time awake, recent sleep quantity and quality, and the person's circadian rhythm. Intrinsic factors ageing [35] and sleep disorders [5]- can increase sleepiness because they impair sleep quantity and quality. Some extrinsic factors -boredom [36], alcohol [37], medication [5], food [38], and time of day [35]- may increase sleepiness further. Other extrinsic factors stimulants as caffeine [33, 39, 40] and nicotine [41, 42]- decrease sleepiness.

\subsection{Classifying and Measuring Sleepiness}

Researchers resolve sleepiness into physiologic-, manifest-, and perceived sleepiness. Physiologic sleepiness is the intensity of the sleep drive at a specific time of day, when external stimuli is minimized. It is the leading accident causative, because physiologic sleepiness depends on previous sleep quantity and quality -or lack of it [4]. Manifest sleepiness is the degree of sleepiness that occurs while interacting with the environment. It sums from the degree of physiologic sleepiness, the degree of environmental stimulation, and the ability to arouse oneself -biased by motivation [4]. Perceived sleepiness is the subjective feeling of sleepiness; biased by physiologic-, environmental-, and personal factors [4]. Measuring any of these requires different approaches. Static posturography is expected to address physiologic sleepiness, because balance is a physiologic function in which the control process does not reach the level of consciousness if the balance situation is consistent with prior experience (reviewed in 2.4.3) [43].

Physiologic sleepiness. The Multiple Sleep Latency Test (MSLT) is quantitative: with electroencephalographic (EEG) measures it assesses the speed of falling asleep in a sleep conductive environment $[44,45]$. The clinical application is wide, but the test is expensive and time consuming. Alternative tests assess physiological markers or biomarkers. Core body temperature [46, 47], melatonin, cortisol [48], and salivary amylase [23] are sensitive to sleep loss. However, these markers are not widely used -perhaps because analyzing 
hormones and enzymes is expensive, testing hormones often is invasive, and reliable temperature testing often relies on rectal thermistors.

Manifest sleepiness. The Maintenance of Wakefulness Test (MWT) measures EEG but assesses -contrary to the $M S L T$ - the ability to resist falling asleep in a comfortable reclining position with eyes closed. The $M W T$ is expensive, time consuming, and difficult to interpret because motivation influences the test results [49]. Alternative tests assess cognitive psychomotor performance, and the available test battery is massive. For instance, the 10-min Psychomotor Vigilance Task $(P V T)$-where the task is to respond to visual cues by pushing a button as quickly as possible, and where the performance is assessed from the number of lapses and reaction time [50, 51]- is common. A specific reaction test that is relatively fast to take and simple to implement is the Critical Flicker Fusion $(C F F)$. Here the subject watches a Light Emitting Diode $(L E D)$ that blinks at decreasing frequencies the task is to push a button when the subject perceives the $L E D$ flicker [33].

Perceived sleepiness. Questionnaires, e.g. the Karolinska Sleepiness Scale (KSS) and the Epworth Sleepiness Scale (ESS) assess subjective sleepiness feelings. In the KSS the subjects rate their alertness on a 9-point scale, ranging from 1 "very alert" to 7 "fighting sleep" [52], whereas in the ESS the subjects use a 4-point scale to rate their tendency to fall asleep in 8 everyday life situations [5]. The questionnaires are rather fast and convenient clinical tools, but because individuals often misjudge their sleepiness, and exhibit varying motivation, the test results are subjective [5]. Sleep diaries are used to record the qualityand quantity of sleep because poor quality (recurrent sleep disruptions) or quantity (less than 7 to $8 \mathrm{~h}$ of sleep per night) cause cumulative sleepiness [5].

Wrapping up, most of the current sleepiness tests are either expensive, time consuming, invasive, subjective, or biased by motivation. This means that the sleep community has a vacancy for a test that addresses these issues - preferably direct testing of physiologic sleepiness, as this factor is the leading accident causative. Because this thesis examines a new approach to evaluate sleepiness, it needs an established method for reference. There are many sleepiness tests to choose from; we employ the $C F F$ test as a reference to posturography because it allows easy realization, testing, and analysis.

\section{BALANCE}

This section reviews balance and factors to take note of when designing a posturographic sleepiness study.

\subsection{Nature of Balance}

Balance is the ability to maintain posture. This thesis refers to balance in quiet standing. Quiet standing appears static, but it is actually dynamic: the sway around the ankle resembles the motion of an inverted pendulum [53]. To maintain balance, balance control must be dynamic for two reasons: (1) The body's mechanical structure is unstable: the 


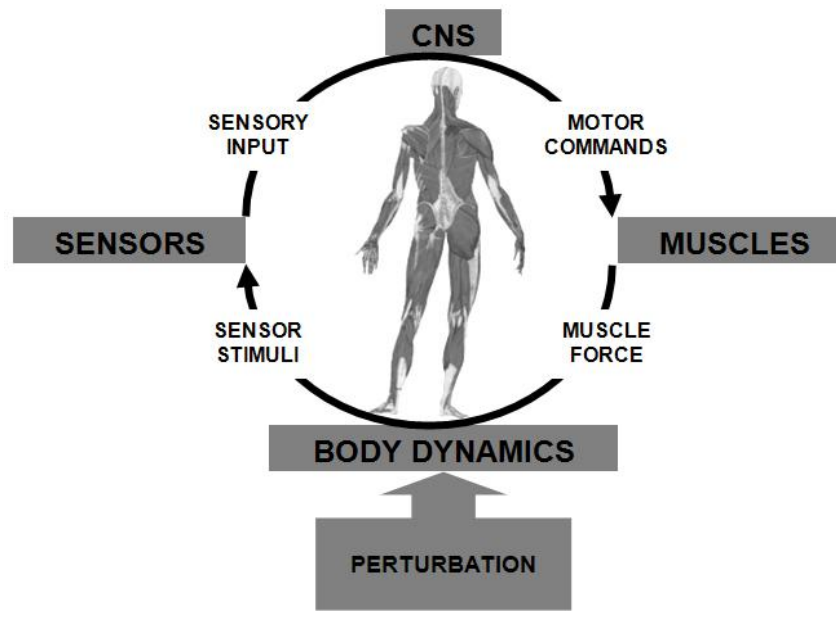

Fig. 1. The feedback control of balance. Figure $4 \mathrm{~b}$ shows the biomechanical interpretation of this process.

Center of Mass $(C o M)$ resides $5 \mathrm{~cm}$ in front of the ankle; the Base of Support $(B o S)$ comprises only two feet, and (2) Heartbeats and breathing perturb the posture continuously. Balance control is a complex closed loop feedback process that the Central Nervous System (CNS) executes (Fig. 1). The CNS maintains the CoM above the $B o S$ by first probing visual-, vestibular-, and proprioceptic sensors for information about the body's current posture, and then by executing appropriate motor commands to the musculoskeletal system [54]. The neuromuscular response to the CoM imbalances generates a Center of Pressure $(\mathrm{CoP})$ trajectory -which is the weighted average of the location of all downward forces acting on the floor through the soles of the feet [55]. The $\mathrm{CoP}$ response depends on the CoM movements and on the motor control of among other muscles, the ankle muscles. The $C o M$ and $C o P$ are virtually in phase (Fig. 4a, right pane), but the $C o P$ must continuously move either ahead or behind the $C o M$ to decelerate it and reverse its direction [55].

\subsubsection{Musculoskeletal System}

The musculoskeletal system comprises the skeleton and postural muscles. The skeleton supports the body. The postural muscles move the skeleton: they attach over joints and contract according to $C N S$ motor commands via motor neurons. Spinal reflexes trigger the fastest muscle responses -in 35 to $45 \mathrm{~ms}-$ and allow regaining balance by regulating muscle contraction and -force [56]. This means, that to record corrective movements the minimum sampling frequency is ca. $30 \mathrm{~Hz}$-but to have at least 3 points during a transition it should be ca. $90 \mathrm{~Hz}$.

In quiet standing primarily the calf- and ankle musculature stabilize the body: this is referred to as ankle strategy [54]. Here contracting tarsal dorsiflexors rotate the skeleton forwards around the ankle joint; contracting tarsal plantarflexors rotate the skeleton backwards. More complex balance situations -e.g. walking- activate knee- and hip musculature: these are referred to as hip- and step strategies [57]. The simplest 
musculoskeletal model is the one-dimensional (1D) single-link inverted pendulum $(S L I P M)$ where a stiff body pivots around the ankle joint in the anteroposterior $(A P)$ direction (reviewed in 2.8). The model relies on the assumption that balance control employs the ankle strategy: during quiet stance the ankle strategy prevails, although the hip strategy is present [58]. The model also relies on the assumption that during quiet stance, $A P$ movements are more prominent than lateral $(M L)$ movements. Quiet stance, with the feet together, produces mainly $A P$ movements, although $M L$ movements are present [58]. Despite the assumptions of the $1 D$ SLIPM, it still applies fairly well to AP sway during quiet stance with the feet together [59]. Modelling situations with hip- and step strategies requires multi-link models $[53,59]$.

\subsubsection{Vestibular, Proprioceptive, and Visual Systems}

The vestibular system in the inner ear detects the acceleration and orientation of the head [60]. Vertical movements stimulate hair cells in the saccule, and evoke impulses in the saccular nerve, whereas horizontal movements stimulate hair cells in the utricle, and evoke impulses in the utricular nerve. Rotational movements stimulate hair cells in the semicircular canals, and evoke impulses in the vestibular nerve. The CNS receives the neural impulses. The mechanical link between head movements and hair cell stimulations is the endolymphatic fluid in the saccule, utricle, and canals. When the head moves the fluid starts to move; the hair cells detect the movement -which is delayed due to the fluid inertia. Factors that impair vestibular function impair balance (reviewed in 2.5.2).

The Proprioceptive System detects the posture of the musculoskeletal system. Skin mechanoreceptors respond to mechanical pressure or distortion under the feet, and muscle proprioceptors respond to muscular movements and detect joint angles [55]. Even if the CNS processes most of this information at an unconscious level, some information is available at a conscious level [43, 61]. Hence, a standing person can affect his/her posture. However, quiet standing with arms crossed over the chest reduces one's ability to affect posture. Moreover, this stance emulates a $1 D$ single-link inverted pendulum.

The Visual System reacts to relative image shifts on the retina. Consequently the system depends on visual acuity and -contrast, object distances, and illumination [62]. At younger years the balance control prioritizes information from the other sensory systems, but with aging the importance of vision in balance control increases [62]. While not crucial for balance control, this means that visual surroundings between consecutive balance tests must be consistent between tests (reviewed in 2.5.2).

\subsubsection{CNS and Balance Control}

The vestibular-, proprioceptic-, and visual information converges in the CNS; especially the cerebellum and brainstem integrate the sensory influx and execute motor control over the postural muscles [43]. To ensure balance control, the sensors operate at partially 
overlapping frequencies [63], but with designated frequency bands. Vision operates below $0.1 \mathrm{~Hz}$, the vestibular system from 0.1 to $0.5 \mathrm{~Hz}$, and the proprioceptive system from 0.5 to $1.0 \mathrm{~Hz}$ [64]. Overlaps may cause conflicting influx to the $C N S$, but the integration process solves any conflicts. The $C N S$ executes the balance control process at an unconscious level in balance situations where the sensory influx is consistent with prior experience [43]. CNS dysfunction or muscular stress shows up as postural tremor between 1 and $3 \mathrm{~Hz}$ [64]. This means that balance data sampled at ca. $30 \mathrm{~Hz}$ or higher needs low pass filtering to allow quantifying the CNS function. Low pass filtering is a standard procedure in physical balance modelling (reviewed in 2.8).

\subsection{Factors Altering Balance}

Balance is not a constant physiologic function and this section reviews factors that affect test results if not accounted for in the study design.

\subsubsection{Sleepiness, Circadian Rhythm, Stress, and Fatigue}

Balance exhibits a circadian rhythm: it has a nadir at night (6-7 am), and in the afternoon (1-4 pm) $[46,65]$. The pattern is consistent with that of the circadian sleepiness: balance correlates with physiologic- [66], manifest- [65], and perceived [64-66] sleepiness. These studies have recorded balance during at least $19 \mathrm{~h}$ of sustained wakefulness, and suggest that sleepiness impairs balance [46, 64-68]. Studies that have recorded balance during $8 \mathrm{~h}$ work shifts support this finding, by reporting that work stress and fatigue impair balance $[8,69]$. These studies have suggested that clinical balance examinations should take into account the time of day. During sustained wakefulness the alertness of the CNS -as measured by pupillography [34]- declines, though the most marked changes occur in the vestibular frequency band [64]. Both changes imply slower response to CoM imbalance.

This means that balance can serve as a physiologic sleepiness marker. It also implies that the time of day for every test should be recorded. Moreover, the low frequency bands involved in the sleepiness related balance changes (CNS 1-3 Hz and vestibular 0.1-0.5 Hz) imply that analyzing the CNS regulated CoM through physical modelling (section 2.8) (rather than the muscular corrections up to $30 \mathrm{~Hz}$ ) may provide an efficient way to quantify sleepiness.

\subsubsection{Intrinsic and Extrinsic Factors}

Ageing impairs balance due to age-related changes in the sensor systems and the postural muscle support. Moreover, the CNS information processing speed gradually slows down [70-72]. Balance disorders -vestibular disorders e.g. Ménière's disease [73], or neurological diseases e.g. Parkinson's disease [74]- impair balance. Also back-, leg-, and muscle disorders and injuries may impair balance. Physically active people generally have 
better balance than average people [75, 76]. Still -the temporary muscle fatigue after physical activities (sport or work) decreases balance [77]. Anatomy and gender influence balance: taller persons sway more than shorter, men sway more than women [72, 78].

Alcohol and drugs (e.g. sleep medication) impair balance [79, 80]. Stimulants, e.g. caffeine, temporarily improve balance [33], although excessive amounts of e.g. caffeine may cause tremor in susceptible people [81]. To allow testing quiet standing -which does not challenge the balance control by stimulating the sensor systems- one must minimize or standardize external stimuli by standardizing room size, illumination, visual cues, noise level, and auditory cues [82]. Standardizing the stance is important because foot placement (BoS area and feet angle) affect balance [78]. The adopted stance also approximates the subject to an inverted pendulum, which facilitates physical modelling of the acquired data (reviewed in 2.8).

\section{POSTUROGRAPHY}

This section reviews the posturographic instrumentation, balance parameterization, and balance modelling of this thesis.

Quantitative posturography quantifies balance in static or dynamic conditions. Static force platform posturography is a non-invasive clinical technique to quantify balance control during quiet standing. The more involved dynamic force platform posturography separates motor-, sensory-, or CNS impairments by challenging the balance control mechanisms with motor-, vestibular-, proprioceptic-, or visual stimuli.

This study employs static force platform posturography which facilitates implementing an instrumented posturographic time awake tester. The reason is that static posturography can detect balance changes related to circadian variations [33, 46, 64-67, 69] and should therefore be able to detect homeostatic changes. Moreover, static posturography is a clinical and commercialized technique -i.e. widely available- and quiet stance is testable on subjects of all ages [72]. Finally, modelling and interpreting quiet stance is easier than modelling and interpreting stance in dynamic test conditions.

\subsection{Force Platform and CoP Trajectories}

This study used a static force platform featuring three force transducers mounted in an isosceles triangle under a circular plate (Fig. 2). The transducers are strain gauges, whose resistance changes in response to pressure, converting applied vertical forces to voltages between -5 and $+5 \mathrm{~V}$. The transducer output $V_{i}$ depends on its gain $G_{i}$. Assuming linearity between transducer input and measured output, the applied vertical force $F_{i}$ satisfies

$$
F_{i}=G_{i}\left(V_{i}-V_{0, i}\right), i=a, b, c,
$$



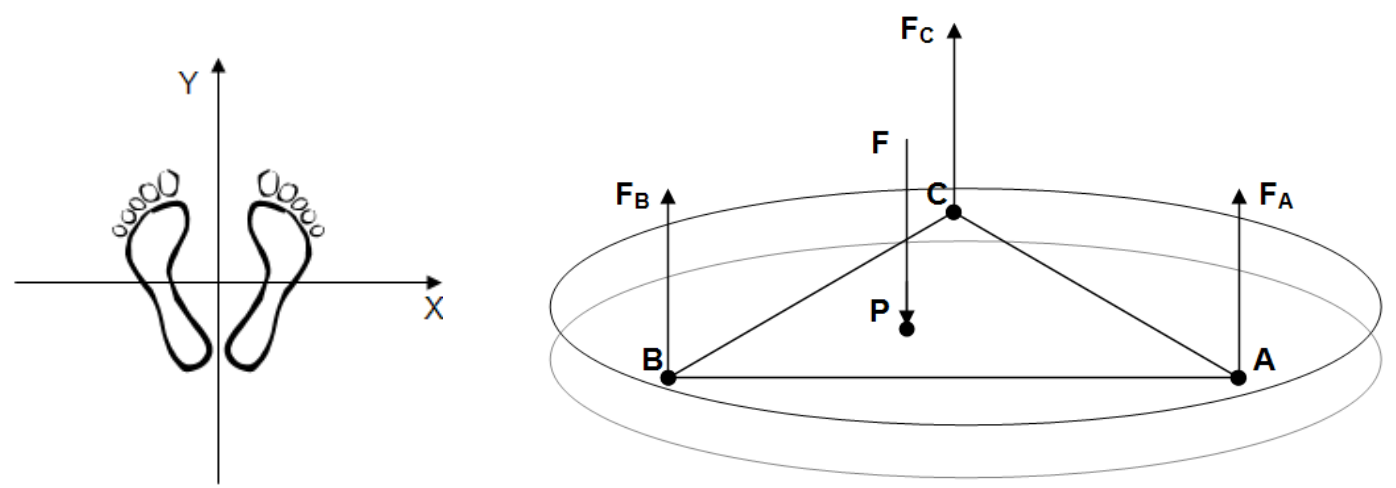

Fig. 2. Left pane shows the force platform coordinate system. The $z$-axis is perpendicular to the $x y$-plane. Right pane shows the mountings of force transducers A, B, and C. P shows the CoP coordinates $\mathbf{p}_{\mathbf{x}}, \mathbf{p}_{\mathbf{y}}$ when a force $F$ acts on the platform. The platform diameter is $43 \mathrm{~cm}$.

where $G_{i}$ is an unknown gain for transducer $i$, and $V_{0, i}$ the transducer's $D C$ offset. Placing a known weight on the platform gives

$$
\mathbf{F}_{\mathrm{a}}+\mathbf{F}_{\mathrm{b}}+\mathbf{F}_{\mathrm{c}}=\mathrm{mg}
$$

where $\mathbf{g}$ is the gravitational acceleration and $\mathrm{m}$ is the weight's mass. By first measuring the transducer offsets, $V_{0}=\left(V_{0, a}, V_{0, b}, V_{0, c}\right)$, and then by measuring a set of transducer outputs, $V_{i}=\left(V_{a, i}, V_{b, i}, V_{c, i}\right) i=1,2, \ldots n,(n \geq 3)$, with the weight at different platform coordinates $i$, Tossavainen et al. [73] define the gain as the solution to

$$
G=V^{\dagger} M, V^{\dagger}=\left(V^{T} V\right)^{-1} V^{T}, V=\left[\begin{array}{c}
V_{1}-V_{0} \\
V_{2}-V_{0} \\
\vdots \\
V_{n}-V_{0}
\end{array}\right], M=\left[\begin{array}{c}
m g \\
m g \\
\vdots \\
m g
\end{array}\right] \text {, and } G=\left[\begin{array}{c}
G_{a} \\
G_{b} \\
G_{c}
\end{array}\right]
$$

During a measurement the transducers register the $C o P$ trajectory -the average of the location of all downward forces, acting on the platform thorough the soles of the standing subject [55] (Fig. 4b). To derive the CoP coordinates $\mathbf{p}_{\mathbf{x}}$ and $\mathbf{p}_{\mathbf{y}}$, we define that for a subject on the platform, the $x$-axis points to the right, the $y$-axis points forward, and the $z$-axis is vertical (Fig. 2). We assume that the platform lies in the $x y$-plane, is weightless and inflexible. Through the (not collinear) transducers at $\mathbf{a}, \mathbf{b}$, and $\mathbf{c}$, the forces $\mathbf{F}_{\mathbf{a}}, \mathbf{F}_{\mathbf{b}}$, and $\mathbf{F}_{\mathbf{c}}$ support the platform (Fig. 2). When a force $\mathbf{F}$ acts on the platform through $\mathbf{p}$, translational and rotational equilibrium require that

$$
\begin{aligned}
& \mathbf{F}_{\mathbf{a}}+\mathbf{F}_{\mathbf{b}}+\mathbf{F}_{\mathbf{c}}+\mathbf{F}=0, \text { and } \\
& \mathbf{a} \times \mathbf{F}_{\mathbf{a}}+\mathbf{b} \times \mathbf{F}_{\mathbf{b}}+\mathbf{c} \times \mathbf{F}_{\mathbf{c}}+\mathbf{p} \times \mathbf{F}=0 .
\end{aligned}
$$


Expanding eq. (2.5) to components gives

$$
\begin{aligned}
& a_{x} F_{a z}+b_{x} F_{b z}+c_{x} F_{c z}=-p_{x} F_{z,} \\
& a_{y} F_{a z}+b_{y} F_{b z}+c_{y} F_{c z}=-p_{y} F_{z} .
\end{aligned}
$$

From eqs. 2.4, 2.6, and 2.7 the vertical forces satisfy [73]

$$
\left[\begin{array}{ccc}
a_{x} & b_{x} & c_{x} \\
a_{y} & b_{y} & c_{y} \\
1 & 1 & 1
\end{array}\right]\left[\begin{array}{l}
F_{a z} \\
F_{b z} \\
F_{c z}
\end{array}\right]=-F_{z}\left[\begin{array}{c}
p_{x} \\
p_{y} \\
1
\end{array}\right],
$$

and when these forces are known, they give $\mathbf{p}$-the CoP trajectory- which the platform records as a function of time.

\subsection{Analyzing $C o P$ Trajectories}

The CoP trajectory during quiet standing is irregular (Fig. 4a). It features the relatively slow CoM movements that the CNS allows, and the relatively fast muscle movements that the corrective motor commands initiate. No consensus on optimal trajectory analysis exists -there are minimum 37 global and structural parameters to choose among [71, 78, 83-85]. Sections 2.7.1 and 2.7.2 review the parameters that this thesis employed (section 4.5 details our parameter selection procedure).

\subsubsection{Global Parameters}

Global parameters -e.g. total sway path, mean velocity, and mean frequency [85]summarize the trajectory size or activity (in time or frequency domains). Generally, declining balance means more sway. The most common sway amplitude $(M C A)$ is

$$
M C A=\frac{\sum_{n} A_{n}}{\sum_{n}}, \text { where } n=F(n) N,
$$

and $F(n)$ is the frequency by which amplitude $A_{n}$ is present in the data, and $N$ is the total number of data points.

\subsubsection{Structural Parameters}

Structural parameters quantify the dynamics or structure of the control process. Short term balance control is positively correlated -open loop- because it preserves the CoP velocity; long term balance control is negatively correlated -closed loop- because it attempts to reverse the $C o P$ velocity [71]. The fractal dimension of sway $(F D)$ of a trajectory 12 


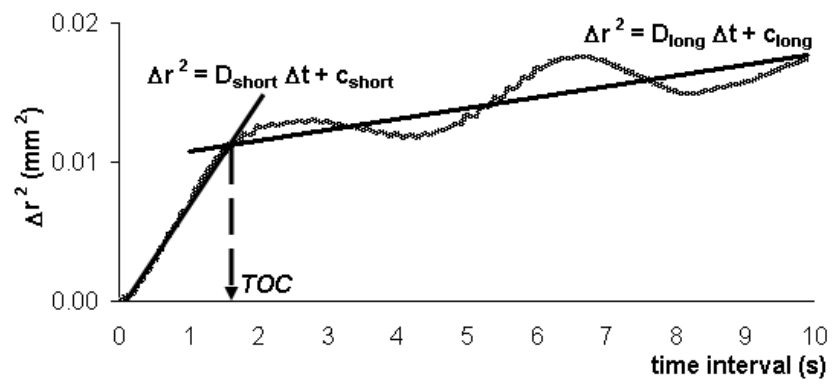

Fig. 3. A diffusion plot with linear regressions in the short term and long term regions. The arrow indicates the TOC. The $y$-coordinate of the critical point is the square planar displacement during the open loop control -the squared distance that $C o P$ travels before it reverses its direction, to decelerate or reverse the direction of the $C o M$ (section 2.4).

quantifies the stochastic activity in the control process. In a given time window $\Delta t$ (spanning $m$ data intervals), a trajectory with $N$ data points is characterized by the following $F D$ [71]

$$
F D=\frac{\log (\Delta r)}{\log (\Delta t)}, \text { where } \Delta r_{\Delta t}^{2}=\frac{1}{N-m} \cdot \sum_{i=1}^{N-m} \Delta r_{i}^{2}, \text { and } \Delta r^{2}=\Delta x^{2}+\Delta y^{2}
$$

In a diffusion plot (Fig. 3) -plotting $\Delta r^{2}$ against $\Delta t$ [71]- the TOC is the critical time interval where open loop control turns into closed loop control. The intersection between a regression in the short term region $\left(\Delta t \leq 0.5 \mathrm{~s}\right.$, slope $D_{\text {short }}$, intersecting $\Delta r^{2}$ in $c_{\text {short }}$ ) and a regression in the long term region $\left(\Delta t \geq 2 \mathrm{~s}\right.$, slope $D_{\text {long }}$, intersecting $\Delta r^{2}$ in $c_{\text {long }}$ ) defines TOC as [71]

$$
T O C=\frac{c_{\text {long }}-c_{\text {short }}}{D_{\text {short }}-D_{\text {long }}}, \text { where } \Delta r^{2}=D \Delta t+c .
$$

\subsection{Physical Models}

Physical models of quiet stance link the $C o P$ trajectory to the $C o M$ movements - which the CNS aims to control $[53,86]$. The single-link inverted pendulum (SLIPM) is widely used hence well documented- to model posture in the $A P$ direction during quiet stance with feet together $[53,55,59,86]$-the stance employed in this thesis. The SLIPM assumes that the balance control employs only the ankle strategy, and that the body sways only in the $A P$ direction. These simplifications mean that the model output rather represents CoM-related movements. However, the agreement between measured and estimated CoM shows that the SLIPM output adequately represents the CoM movements [59].

The SLIPM treats the upright body as an inverted pendulum pivoting around the ankle (Fig. 4b). The model assumes that the vertical sway angle $\theta$ is small, it ignores the horizontal forces acting on the soles of the feet, and it ignores the weight of the feet. The sum of torques acting on the pendulum relates the CoM displacement $y_{g}$ to the CoP displacement $y_{p}$ as [53] 


$$
m g\left(y_{p}-y_{g}\right)=I \alpha
$$

where $m$ is the body weight concentrated in the CoM, $I=m h^{2}$ is the moment of inertia for a $\operatorname{CoM}$ at height $h$. For a small vertical sway angle $\theta$, the angular acceleration $\alpha$ approximates to $-\ddot{y}_{g} / h$. By substituting $I$ and $\alpha$ into eq. 2.12 , the relation becomes [53]

$$
y_{p}=y_{g}-\frac{h}{g} \ddot{y}_{g} .
$$

Laplace transforming eq. 13 gives

$$
Y_{P}(s)=\left(1-\frac{h}{g} s^{2}\right) Y_{G}(s)
$$

and

$$
Y_{G}(s)=\frac{g / h}{g / h-s^{2}} Y_{P}(s)=\frac{\sqrt{g / h}}{2}\left(\frac{1}{s+\sqrt{g / h}}-\frac{1}{s-\sqrt{g / h}}\right) Y_{P}(s),
$$

using partial fractions. The convolution * inverts eq. 15 and gives

$$
y_{g}(t)=\frac{\sqrt{g / h}}{2} e^{-|t| \sqrt{g / h}} * y_{p}(t) .
$$

Discretizing eq. 2.16 with respect to time gives [73]

$$
\hat{y}_{g}(n)=\frac{T \sqrt{g / h}}{2} e^{-|n T| \sqrt{g / h}} * y_{p}(n),
$$

where $T$ is the sampling interval. This study used eq. 2.17 to extract $y_{g}(t)$ from $y_{p}(t)$. Figure $4 \mathrm{a}$ (right pane) exemplifies $y_{g}(t)$ versus $y_{p}(t)$. Figure $4 \mathrm{~b}$ relates $y_{g}(t)$ and $y_{p}(t)$ of eq. 2.12 to a biomechanical model of the neural balance control process. 

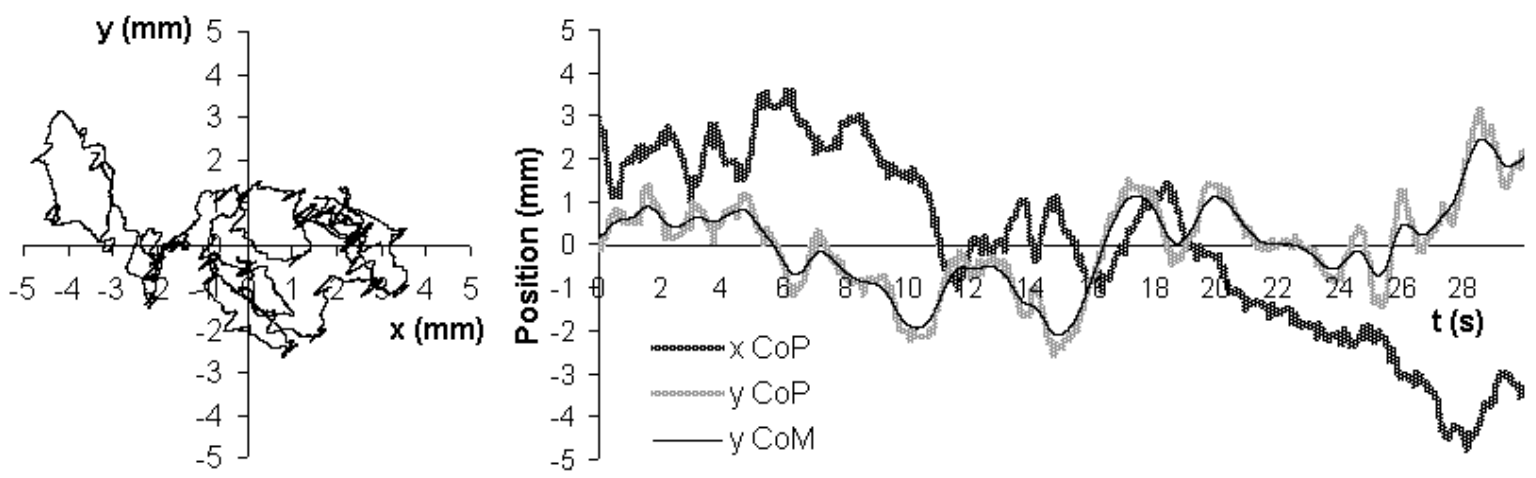

Fig. 4a. Left pane shows a $C o P$ trajectory during quiet stance: lateral sway plotted against anteroposterior sway ( $x$-axis points to the right, $y$-axis forward). Right pane shows $x$ and $y$ of $C o P$, and $y$ of $C o M$ during a posturographic test.

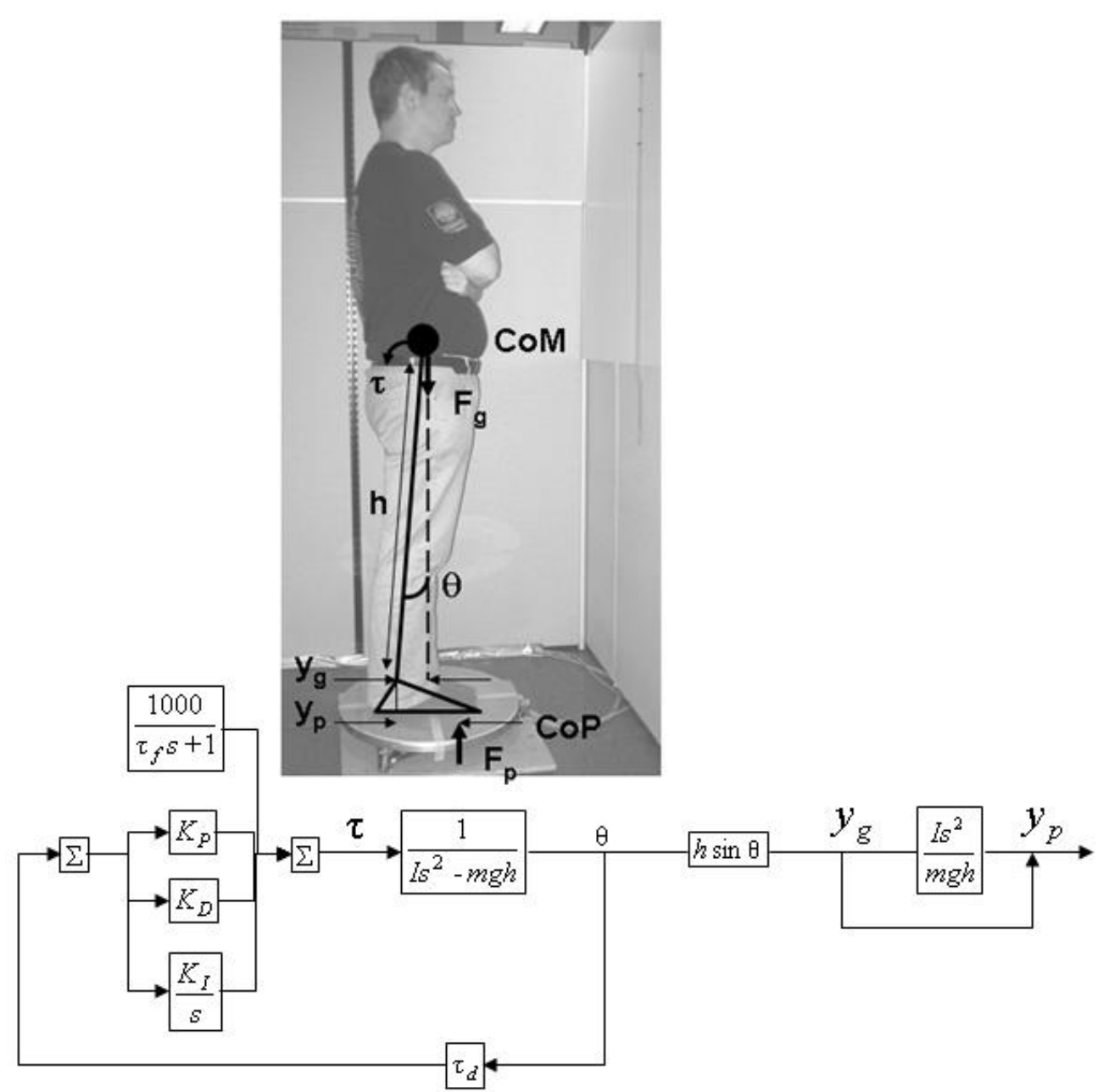

Fig. 4b. The vector diagram shows the SLIPM during quiet standing: the forces and torques that act on the pendulum. The SLIPM ignores the weight of the feet and the horizontal forces acting on them. The control diagram shows a biomechanical model that interprets closed loop feedback control during quiet standing (section 2.4, Fig. 1). The neural control components that affect the $y_{p}$ and $y_{g}$. of the SLIPM (eq. 2.12) are: $\tau_{d}$ relating to time delay of the system, $\mathrm{K}_{P}$ relating to stiffness-, $\mathrm{K}_{D}$ relating to damping-, and $\mathrm{K}_{I}$ relating to error control of the system. The diagram is adapted from Peterka 2000 [86] and is shown to facilitate later discussion on balance control in section 6.3 . 


\subsection{Validity and Repeatability of Posturography}

Static posturography identifies balance changes related to normal ageing [71, 72] and circadian variations [33, 46, 64-69]. Moreover, generally the daily [87], weekly [88], and monthly [88] test-retest reliability of static posturography is reported to be high, -but there are reports on low reliability [89]. The discrepancy between reported reliability may stem from the differences in test protocols and study populations. However, the force platform technique is widely used as a clinical tool to assess balance. The technique shows promise as a predictor of falls among elderly [90], as a diagnostic tool for balance deficits [73, 74], and also for quantifying the harmful effects of alcohol and solvents [79, 80, 91].

Because static posturography identifies the circadian component, it should be able to identify the homeostatic component of sleepiness -if the balance exhibits it. The veracity of static posturography -identifying age-related and pathology-related balance changesmeans that static posturography is a valid balance test. The test-retest reliability means that the method results are repeatable. Summing up, posturographic time awake testing should be valid and precise.

\subsection{Balance Testing During Sustained Wakefulness}

Fatigue-related balance deficits are well documented; postural sway increases due to declining balance during 5 to $8 \mathrm{~h}$ work shifts $[8,69]$. Studies that have monitored balance during sustained wakefulness -minimum $19 \mathrm{~h}$ - show that balance varies with the time of day [33, 46, 64-68]. These studies based their findings on 15 to $120 \mathrm{~s}$ trials where balance was sampled at 20 to $50 \mathrm{~Hz}$-most common was a $30 \mathrm{~s}$ trial and a $32 \mathrm{~Hz}$ sampling rate.

\section{SYNTHESIZING A POSTUROGRAPHIC TIME AWAKE TESTER}

This section aggregates the factors that support setting up a posturographic time awake tester feasible for estimating acute sleep deprivation, and the resulting sleepiness. The section also gives the guidelines to validate the tester.

Four factors support the hypothesis that time awake is posturographically estimable. First, posturography is sensitive, -it quantifies balance impairments related to normal ageing. Second, posturography is repeatable, -its test-retest reliability is high. Third, balance is circadian, -posturography is sensitive to circadian balance variations. Moreover, since posturography quantifies the circadian component, posturography could also quantify the homeostatic component, -provided balance responds to sleep homeostasis. This possibility is important, because the homeostatic component depends on time awake. Fourth, balance is a physiologic function, -hence it is not overly affected by factors that bias cognitive sleepiness tests.

The circadian feature in balance affects the design of posturographic time awake testing: during sustained wakefulness, a circadian balance improvement could return balance close 
to the level prevalent when the wakefulness begun. If the tester does not account for this time of day effect, it could misinterpret the magnitude of the homeostatic component, and hence -the current time awake. Moreover, the circadian phase and amplitude are personal [29] -hence, posturography -similarly to existing sleepiness tests- applies to individual testing $[64,88]$. To examine the feasibility of employing posturography for sleepiness testing it is thus necessary to:

1. Test balance with $30 \mathrm{~s}$ trials every $2 \mathrm{~h}$ during $36 \mathrm{~h}$ of sustained wakefulness. This ensures recording balance during a full circadian period.

2. Test sleepiness with a known reference method -e.g. the $C F F$. This ensures recognizing momentary balance improvements by comparison with the $C F F$ scores, and allows deconvolution of the diurnal balance scores.

3. Focus the data analysis on finding balance parameters (global, structural, or model) whose monotone correlation with time awake (the homeostatic component) explains the diurnal variance of balance.

4. Test balance and estimate the current time awake at each test, by equating the balance score with those tabulated in a personal reference table (balance scores during sustained wakefulness tabulated against time awake and local time, item 1 above). This ensures that personal circadian phases and amplitudes do not bias the estimate.

5. Evaluate the effect of test length on the accuracy of the time awake estimates.

6. Evaluate whether a population mean of balance scores recorded at 13:30 hours -the general afternoon sleepiness peak- could serve as a threshold to identify excessive sleepiness in individuals.

Generally, researchers employ posturography to assess age-, health-, stimulant-, alcohol-, or drug related effects on balance. However, during development and validation of a posturographic time awake tester one should aim to exclude several factors that affect sleepiness and balance, -e.g. age, health, caffeine, alcohol, and drugs. Hence, a study design that conforms to literature guidelines should:

1. Select healthy persons from a homogeneous age- and socioeconomic group, and exclude smokers. 
2. Prohibit alcohol $24 \mathrm{~h}$ prior to any testing and caffeine $12 \mathrm{~h}$ prior to any testing (both prohibited during testing).

3. Standardize the environment; offer meal intakes promptly after a test, and offer standardized activities.

4. Record the time of day of any test and use each subject as his/her own (sleepinessand balance) reference.

5. Standardize the balance test: e.g. unshod quiet standing with feet together and with arms crossed over chest, looking at a fix point provided for visual reference.

6. Test balance with $30 \mathrm{~s}$ trials and sample the $C o P$ traces with at least $33 \mathrm{~Hz}$.

7. Standardize the test room; minimum $3 \mathrm{~m} \times 4 \mathrm{~m}$ with 'normal' 500 lx illumination, 'low' $40 \mathrm{~dB}$ sound, and test balance at the room center to eliminate auditory cues, e.g. echoes from the walls.

\section{STUDY AIM}

This study examines posturographic time awake testing for instrumentation purposes -to set the scientific foundation for a time awake tester. The specific study aims ${ }^{1}$ are to:

1. Quantify monotone balance impairment, and $C F F$ changes, during increasing time awake (I).

2. Estimate time awake with posturographic balance tests (II).

3. Quantify longitudinal repeatability of balance tests, and daytime changes in balance (III).

4. Validate posturographic time awake testing (IV).

5. Quantify tester efficiency: estimation accuracy versus test length (V).

6. Improve the validity of the posturographic tester by modelling balance as an inverted pendulum (VI).

\footnotetext{
${ }^{1}$ The Roman numerals indicate the paper that mainly dealt with the listed aim. 


\section{SUBJECTS AND METHODS}

\subsection{Subjects}

We tested 63 subjects on three occasions within the frame of this thesis. Table 1 shows the subject characteristics. All subjects were volunteers (students and staff) at the University of Helsinki. We assessed the subjects' health with a questionnaire; exclusion criteria were smoking, diagnosed balance- or sleep disorders, current leg- or back injuries, or current medication influencing sleepiness. Each subject gave their written informed consent before inclusion in the study.

Table 1. Subject characteristics ${ }^{\dagger}$ in studies I-VI.

\begin{tabular}{|c|c|c|c|c|}
\hline & \multicolumn{4}{|c|}{ Study } \\
\hline & I, II, V & III & $\mathrm{IV}, \mathrm{VI}$ & Total \\
\hline $\mathrm{N}(\text { men/women })^{\dagger \dagger}$ & $21(17 / 4)$ & $30(15 / 15)$ & $12(10 / 2)$ & $63(42 / 21)$ \\
\hline Age (yr) & $28(20-37)$ & $27(23-37)$ & $27(21-38)$ & $27(20-38)$ \\
\hline Height (m) & $1.77(0.08)$ & $1.74(0.07)$ & $1.78(0.05)$ & $1.76(0.07)$ \\
\hline Weight (kg) & 70 (13) & $74(11)$ & $76(15)$ & $73(12)$ \\
\hline Exercise (h/week) & $2.0(1.3)$ & $2.8(1.5)$ & $2.6(3.0)$ & $2.5(2.0)$ \\
\hline
\end{tabular}

\subsection{Study Designs}

Alcohol was prohibited $24 \mathrm{~h}$ prior to any testing, whereas caffeine was prohibited $12 \mathrm{~h}$ prior to any testing. Exercise was prohibited during sustained wakefulness conditions.

Study I was methodological, designed to quantify balance during sustained wakefulness. First, the subjects took a 'single' balance test, reported their current time awake, and practiced $C F F$ testing. Second, the subjects were kept awake from 8:00 hours for at least $28 \mathrm{~h}$, during which we tested their balance and $C F F$ every $2 \mathrm{~h}$. At the first 8:00 hours test, the subjects took the test five times. Third, we extracted the monotone balance impairment from the diurnal balance variation. Fourth, using 6 training set subjects, we screened among 163 balance parameters for parameters whose linear correlation between balance scores and time awake accounted for the highest percentage of the variance. Fifth, for those balance scores we calculated, using 15 test set subjects, the 'accuracy' of the time awake estimate as the time interval needed to elapse before it separated two consecutive balance scores with $95 \%$ probability.

Study II was also methodological, designed to estimate time awake with posturographic balance tests. Study I provided our database. First, we regressed the balance scores from the sustained wakefulness condition on time awake. Second, we estimated the subject's time awake from the regression plot by equating the balance score from the single test with 
those recorded during the sustained wakefulness. Third, if this resulted in more than one candidate estimate -due to diurnal variation- we selected the estimate whose local time instant was closest to the local time instant of the single test.

Study III was longitudinal, designed to quantify repeatability across a month, and impairment during daytime, of the balance scores used to estimate time awake. First, we tested balance once a week during one month -on the same weekday at 8:30 hours the subjects' took one test. Second, we tested balance at 8:30, 10:30, and 13:30 hours -the subjects' took one test per time point. Longitudinal repeatability was evaluated regressing the balance scores on the test weeks, whereas daytime changes were evaluated regressing the balance scores on daytime.

Study IV was designed to validate studies I-III. First, the subjects were kept awake from 8:00 hours for $36 \mathrm{~h}$, during which we tested their balance every $2 \mathrm{~h}$. At the first 8:00 hours test, the subjects took the test five times. Second, we tested their balance once a day during one week; each day with different and randomly assigned time awake. We assured spread in a subject's 7 time awake lengths by scheduling each test's time awake to differ at least $2.5 \mathrm{~h}$ from all previous time awakes. Third, we tested their balance once a week during one month: same weekday, local time, and time awake. We estimated time awake at each daily balance test as in Study II, and evaluated the accuracy and precision of the estimates across days and weeks.

Study V was analytical, designed to define how the accuracy of a time awake estimate depends on the test length. Study I provided the database. First, using $30 \mathrm{~s}$ tests, we extracted the monotone balance impairment from the diurnal balance variation, regressed balance scores on time awake, and calculated their time awake estimation accuracy -as in Study I. Second, we recalculated the accuracy with successively shorter balance tests truncating the $30 \mathrm{~s}$ data second-by-second from the end. Third, we regressed the accuracy against test length.

Study VI was analytical, designed to improve the validity of the time awake tester by modelling balance as an inverted pendulum. Study IV provided the database. First, we extracted the CoM-related movements from the CoP trajectories with the SLIPM. Second, we estimated time awake at each daily balance test as in Study II, and evaluated the accuracy and precision of the estimates across days and weeks.

In studies I-V we did not presume a balance model -we used global and structural parameters to directly quantify the $A P$ - and $M L C o P$ trajectories. In Study VI we employed the single-link inverted pendulum model to link the $A P$ direction of the $C o P$ trajectory to the $A P$ direction of the $C o M$-related movements. The $C o M$-related movements were then quantified with the same parameters as in studies I-V.

\subsection{Balance Testing and Platform Functioning}

Balance was tested with a force platform on which the subject stood: unshod, feet together, arms crossed over chest, looking at an eye-high fix point in front of the platform (Fig. 5). 20 
In studies I and IV the platform sampled body $C o P$ excursions at $1 \mathrm{kHz}$ for $30 \mathrm{~s}$ : the data was filtered with a $0.3 \mathrm{~s}$ moving average. In Study III the platform sampled and filtered the data at $33 \mathrm{~Hz}$ for $22 \mathrm{~s}$. We calculated the balance scores FD, MCA, and TOC (for Studies I$\mathrm{V}$ ), and the $C o M$-related movements $y_{g}(t)$ (for Study VI), from the filtered $C o P$ traces.

We ensured each morning that the output of the force transducers had not drifted. If the output of any of the transducers had drifted more than 5\% from the study onset, we recalibrated the transducers with a $20 \mathrm{~kg}$ weight according to eq. 2.3 in section 2.6. This criterion rendered calibrations unnecessary during the study.

\subsection{CFF Testing}

We employed a $C F F$ device (Fig. 5) as a reference to the posturographic test. The subject wore hearing protectors and sat, chin supported, $30 \mathrm{~cm}$ in front of a red $L E D(\varnothing 5 \mathrm{~mm})$. One test comprised 18 sequences, with a 1 min rest after every $6^{\text {th }}$ sequence. During one sequence the $L E D$ blinked at decreasing frequencies from 40 to $30 \mathrm{~Hz}$ in $10 \mathrm{~s}$. The subject watched the $L E D$ and pressed a button when perceiving the $L E D$ flicker. The $C F F$ score in $\mathrm{Hz}$ was the $30 \%$ trimmed average of the 18 button pushes.

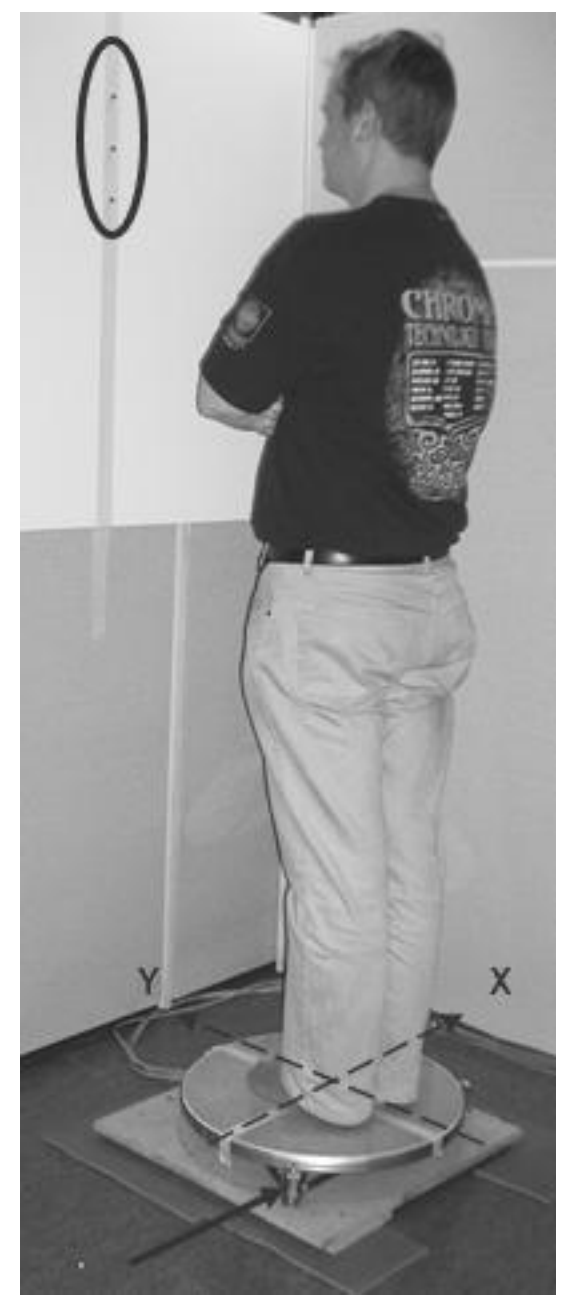

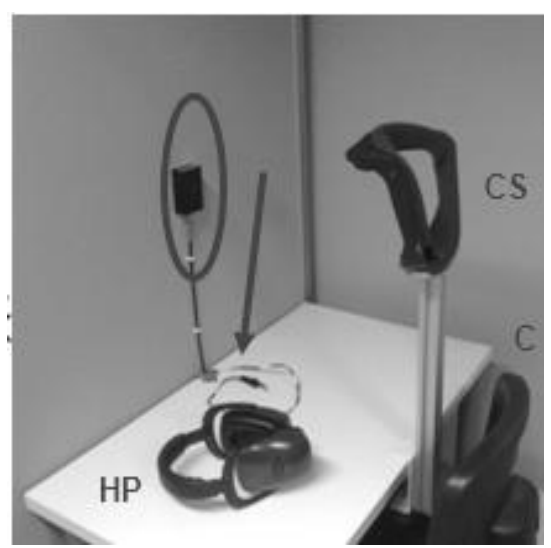

Fig. 5. Left pane shows balance testing with a force platform, and the coordinate system of the platform. The oval indicates eye-high fix points. The arrow indicates force transducer "B" in Fig. 2. Right pane shows the CFF tester, hearing protectors (HP), chin support (CS), and adjustable chair (C). The oval indicates $L E D$, The arrow indicates a push button. 


\subsection{Quantifying Monotone Balance Impairments (Study I)}

Each subject took a balance- and $C F F$ test every $2 \mathrm{~h}$ during at least $28 \mathrm{~h}$ of sustained wakefulness. First, we presented balance parameter no. $i\left(i=[1,163]^{2}\right)$ during sustained wakefulness $t(t=[2, \max t])$ as $P_{i}(t)$ (Fig. 6a). Second, we extracted the monotone, time awake dependent balance impairment by deconvolving -i.e. element-by-element vector division- $P_{i}(t)$ with the $C F F(t)$ scores (Fig. 6c):

$$
P_{i}^{d}(t)=P_{i}(t) / C F F(t)
$$

Third, we regressed each $P_{i}^{d}$ against $t$,

$$
\hat{P_{i}^{d}}=a+b \cdot t
$$

and calculated the linear correlation $R_{i}^{2}$ between $P_{i}^{d}$ and $t$. Fourth, among all correlations $\left[R_{1}^{2}, R_{163}^{2}\right]$, we screened for the three highest correlations $\left[R_{A}^{2}, R_{B}^{2}, R_{C}^{2}\right]$ and denoted the respective parameters $\left[P_{A}, P_{B}, P_{C}\right]$ as the subject's personal parameters. Fifth, we screened among the training set subjects, and identified the personal parameters showing the three highest fraction of incidences (\%) within the set. We denoted these parameters as selected parameters. Thus, the selected parameters are common parameters that have a linear correlation between balance scores and time awake which account for the highest percentage of the variance in balance. The successive work of this thesis deals only with the selected parameters (Table 2). Sixth, for the selected parameters we calculated the 'accuracy' of the time awake estimate as the time interval needed to elapse before it separated two consecutive balance scores with $95 \%$ probability:

$$
\Delta T^{P_{i}}=\hat{P_{i}^{d}}(\max t) / 2 \cdot t_{0.025} \cdot \sqrt{\frac{1}{n-2} \cdot \frac{\sum\left(P_{i}^{d}-\hat{P_{i}^{d}}\right)^{2}}{\sum(t-\bar{t})^{2}}},
$$

where $n$ was the number of balance tests and $t_{0.025}=2.14$ [92].

\footnotetext{
${ }^{2}$ Collected from the posturographic literature [70, 77, 82-84], and from the signal processing literature (available from the thesis's author). 


\subsection{Estimating Time Awake (Study II)}

First, we plotted a reference curve based on the reference table: balance scores from the sustained wakefulness condition regressed against time awake, and against local time. The standard deviation $(S D)$ of the 5 test repetitions at 8:00 hours provided the error bars of the reference curve. Second, we equated the score from the single test with the scores in the reference curve. The time awake instant(s) where the single score equaled the reference score(s), was registered as the estimate candidate(s). Third, we accepted the candidate estimate whose local time was closest to the local time of the single test (Fig. 7). An estimate within $\pm 2.5 \mathrm{~h}$ of the true time awake (subject's information) was defined 'successful' to allow calculating the predictive values of the time awake tester.

\subsection{Quantifying Repeatability and Daytime Changes (Study III)}

Longitudinal repeatability was quantified by testing each subject's balance once a week during a month: same weekday, same local time. ANOVA (SPSS v. 11.0) with repeated measurements for test week rejected the hypothesis of test repeatability across the month, if the test weeks differed significantly from each other, i.e. if $P \leq 0.05$.

Daytime changes were quantified by testing each subject at 8:30, 10:30, and 13:30 hours on the same day. Linear regression of balance against daytime quantified (the intercept $a$, and slope $b$, of the regression line) the daytime dependency. ANOVA with repeated measures for time indicated significant daytime changes, if $P \leq 0.05$.

\subsection{Validating Posturographic Time Awake Testing (Study IV)}

Each subject took a balance test every $2 \mathrm{~h}$ during $36 \mathrm{~h}$ of sustained wakefulness to generate his/her reference curve, and the $S D$ of the 5 test repetitions at 8:00 hours provided the error bars of the reference curve. Each subject also took a single test once a day during one week (Test set) and a single test once a week during one month (Longitudinal test set).

Validity was quantified by first estimating time awake as in Study II, and then by comparing the estimate to the true time awake -the subject's self-report. An estimate within $\pm 2.5 \mathrm{~h}$ of the true time awake was defined 'positive' to allow calculating the accuracy of the time awake tester.

Precision of the estimates was quantified as (1-SD/mean) $\times 100 \%$ : 'high precision' required precision $>95 \%$.

Longitudinal trends in the estimates were quantified by regressing daily accuracies against test day: if the Pearson correlation was significant, i.e. $P \leq 0.05$, it implied trends across the sets. 


\subsection{Quantifying Test Accuracy Versus Test Length (V)}

For all $j=[2,30] \mathrm{s}$ of test data we used eqs. 4.1 to 4.3 to calculate the 'accuracy' $\Delta T^{P_{j}}$; the time interval needed to elapse before it separated two consecutive test scores with $95 \%$ probability. Here $P$ was a selected parameter (defined in section 4.5 for Study I). Linear regression of mean $\Delta T^{P}$ against $j$ implied test length dependency, if the Pearson correlation was significant, i.e. $P \leq 0.05$. The shortest test length that separated (with $95 \%$ probability) two consecutive test scores when $5 \mathrm{~h}$ had elapsed was defined $j_{\min }$.

\subsection{Improving Test Validity (Study VI)}

Here we used the SLIPM to model balance control during sustained wakefulness. First, we used eq. 2.17 to extract the $C o M$-related movements $y_{g}(t)$ from the $A P C o P$ trajectories $y_{p}(t)$. Second, we quantified $y_{g}(t)$ with the selected parameters. Finally, we evaluated the validity of the posturographic tester as in Study IV. We defined heuristically a 5\% increase in accuracy and a 5\% increase in precision as improvement.

\section{MAIN RESULTS}

\subsection{Monotone Balance Impairments (Study I)}

The training set aimed at identifying (by subject-wise screening) the three balance parameters whose linear correlation with time awake were the highest. Figure 6 shows a case study. Regression analysis in the sustained wakefulness condition revealed a linear correlation between balance and time awake that accounted for $55 \%$ of the balance variance (Fig. 6a), whereas the monotone time awake dependent balance impairment (eq. 4.2) revealed a linear correlation that accounted for $88 \%$ of the balance variance (Fig. 6c). Figure 6 also shows the correspondence between the posturographic- and $C F F$ test results. Among the available balance parameters, the $F D$ (eq. 2.10) exhibited the highest time awake correlation for three out of six subjects, whereas the $M C A$ (eq. 2.9) exhibited the highest time awake correlation for two out of six subjects, and the TOC (eq. 2.11) exhibited the highest time awake correlation for three out of six subjects (bolded in Table 2, left pane). We denoted the $F D, M C A$, and $T O C$ as selected parameters. Table 2 (right pane) summarizes the group mean of the linear correlation between the selected parameters and time awake, and also shows their group mean 'accuracy' (eq. 4.3).

The test set subjects showed that the 'accuracy' (eq. 4.3) of the FD, MCA, and TOC parameters was better than $5 \mathrm{~h}$ in $80 \%$ of the cases (Table 3). When selecting the best achievable accuracy per subject (bolded in Table 3), the group mean accuracy was $3.0 \pm 3.1$ $\mathrm{h}$ (neither $0.0 \mathrm{~h}$ nor $-0.1 \mathrm{~h}$ imply infinite or negative accuracy for any subject; the large range stems from the result of subject $\mathrm{N}$, cf. Table 3 ). 
This means that we identified the balance parameters most responsive to increasing homeostatic sleep drive, and that we validated the posturographic test against the $C F F$ test.

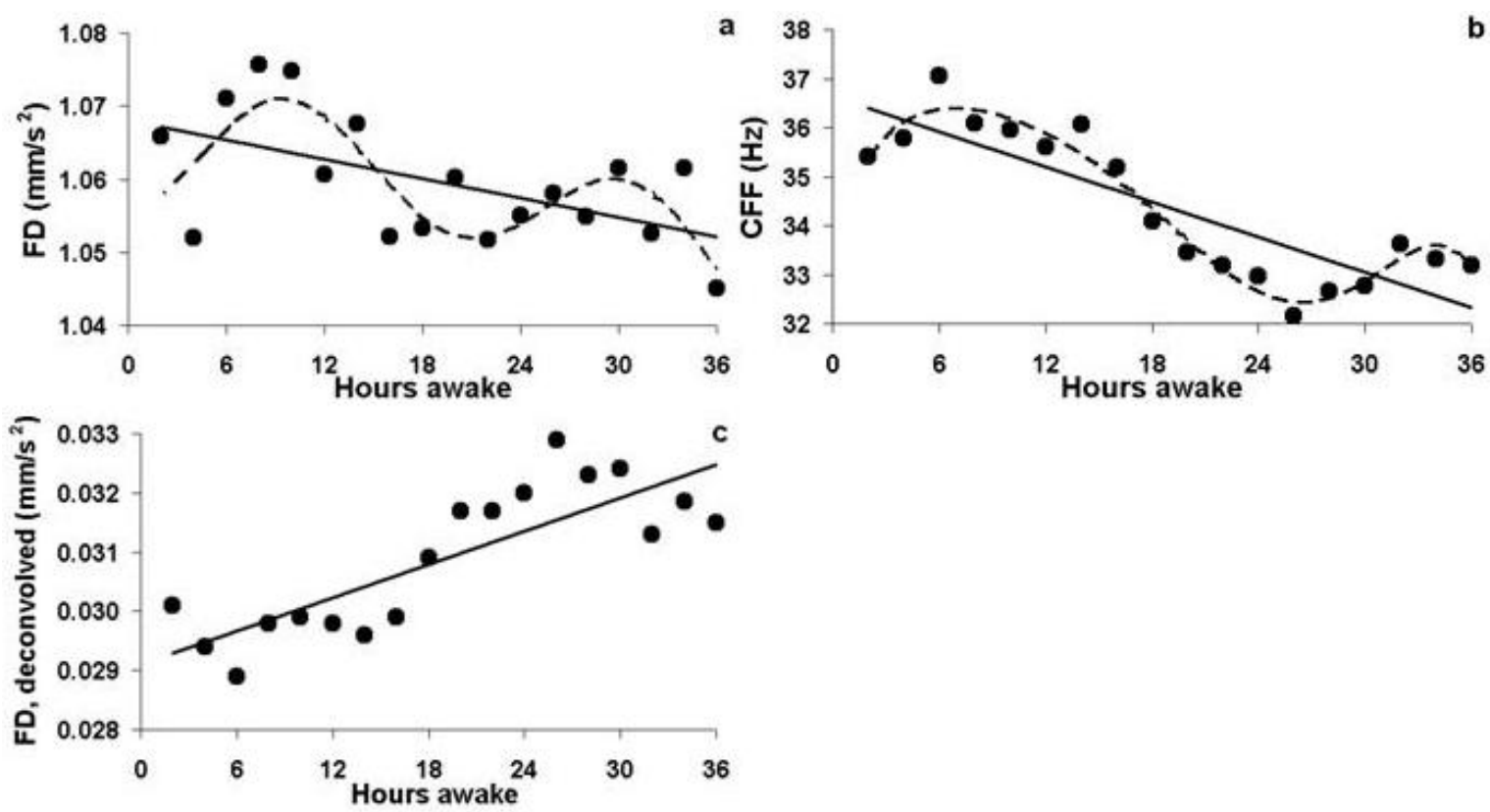

Fig. 6. Scatter plot and linear regression of test scores (balance expressed as $F D$ ) against time awake for: a) $F D\left(F=7.03, P=0.017, R^{2}=0.55\right)$, b) $C F F\left(F=40.7, P<0.0001, R^{2}=0.85\right)$, and c) deconvolved $F D(F=33.7$, $P<0.0001, R^{2}=0.88$ ). The solid lines indicate the linear regression, the dashed lines facilitates visual inspection of the circadian rhythm. Two hours awake corresponds to 8:00 hours local time. This figure shows the time awake dependent balance impairment, and the correlation between the posturographic- and CFF test results.

Table 2. Left pane shows subject-wise the balance parameters (unitless numerals) that exhibited the highest linear correlation with time awake. The selected parameters (bolded numerals) exhibited the highest correlation for up to $50 \%$ of the subjects (i.e. $50 \%$ for $P_{14}$, the $F D, 33 \%$ for $P_{65}$, the $M C A$, and $50 \%$ for $P_{143}$, the TOC). Right pane shows the characteristics of the selected parameters: linear correlation with time awake, $R^{2}$, and time awake estimation accuracy.

\begin{tabular}{|c|c|c|c|c|c|c|c|c|c|}
\hline \multirow{2}{*}{$\begin{array}{l}\text { Balance } \\
\text { parameters }\end{array}$} & \multicolumn{6}{|c|}{ Subject } & \multirow{2}{*}{$\begin{array}{l}\text { Selected } \\
\text { parameters }\end{array}$} & \multicolumn{2}{|r|}{$\overline{\text { Mean }}$} \\
\hline & $\overline{\mathrm{A}}$ & $\bar{B}$ & $\bar{C}$ & $\bar{D}$ & $\bar{E}$ & $\bar{F}$ & & $\overline{R^{2}}$ & Accuracy (h) \\
\hline$\overline{P_{A}}$ & 65 & 17 & 14 & 14 & 14 & 22 & $P_{14}(F D)$ & 0.53 & 2.8 \\
\hline$P_{B}$ & 124 & 53 & 136 & 63 & 65 & 36 & $P_{65}(M C A)$ & 0.81 & 2.5 \\
\hline$P_{C}$ & 143 & 143 & 143 & 100 & 152 & 69 & $P_{143}(T O C)$ & 0.63 & 2.6 \\
\hline
\end{tabular}


Table 3. Subjects G-U: time awake estimation accuracy, in hours, of selected balance parameters individual and group mean. Bolded accuracies identify the subject's best achievable accuracy. This table shows the individual accuracy ranges of the selected parameters.

\begin{tabular}{|c|c|c|c|c|c|c|c|c|c|}
\hline \multirow{2}{*}{$\begin{array}{l}\text { Selected } \\
\text { parameters }\end{array}$} & \multicolumn{9}{|c|}{ Subject } \\
\hline & G & $\mathrm{H}$ & I & $\mathrm{J}$ & K & $\mathrm{L}$ & $\mathrm{M}$ & $\mathrm{N}$ & $\mathrm{O}$ \\
\hline$\overline{F D}$ & 10.1 & 0.1 & 10.3 & $\overline{0.1}$ & 10.7 & 18.4 & 3.9 & 13.1 & 14.2 \\
\hline MCA & 3.2 & 9.3 & 6.3 & 8.7 & 0.2 & 5.9 & 1.6 & 21.2 & 3.7 \\
\hline \multirow[t]{3}{*}{ TOC } & 17.5 & 1.7 & 15.8 & 7.9 & 6.7 & 3.4 & 3.5 & 11.5 & 4.3 \\
\hline & \multicolumn{9}{|c|}{ Subject Continued } \\
\hline & $\mathrm{P}$ & Q & $\mathrm{R}$ & S & $\mathrm{T}$ & $\mathrm{U}$ & \multicolumn{3}{|c|}{$\operatorname{Mean}(\mathrm{SD})$} \\
\hline FD & 4.1 & 2.6 & 1.6 & 8.7 & 3.7 & 31.3 & \multicolumn{3}{|c|}{$8.9(8.2)$} \\
\hline MCA & 5.1 & 4.9 & 6.7 & 5.7 & 2.1 & 4.5 & \multicolumn{3}{|c|}{$5.9(4.8)$} \\
\hline TOC & 0.5 & 10.1 & 5.9 & 5.4 & 0.1 & 10.1 & \multicolumn{3}{|c|}{$6.9(5.2)$} \\
\hline
\end{tabular}

\subsection{Time Awake Estimates (Study II)}

The training set showed that between the $2^{\text {nd }}$ and $30^{\text {th }}$ hour awake balance scores impaired by $0.68 \%$ per hour; declining balance increases the TOC [70]. Regression analysis in the sustained wakefulness condition revealed a linear correlation between balance and time awake that accounted for roughly $60 \%$ of the balance variance (Fig. 7).

The test set showed that regressing the estimated time awake against true time awake showed a significant $(P<0.0001)$ linear correlation, that accounted for roughly $85 \%$ of the variance (Fig. 8). For each 1-h increase in true time awake, the estimated time awake increased by $1.02 \mathrm{~h}$. The positive predictive value of the posturographic time awake estimate was $69.2 \%$, the negative predictive value $93.3 \%$, the sensitivity $56.3 \%$, and the specificity $96.0 \%$.

This shows that the proposed time awake estimation procedure is a promising concept. 

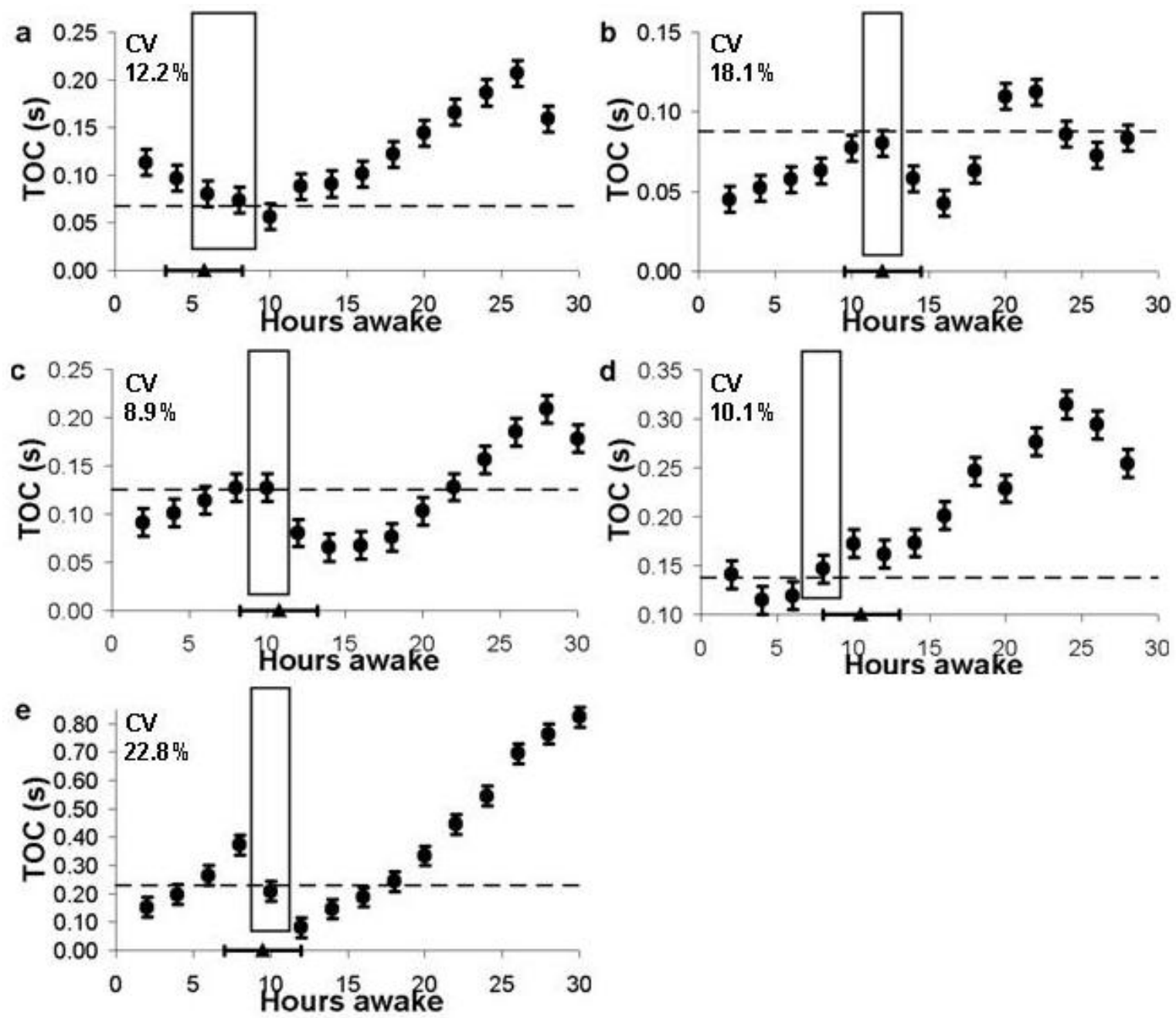

Fig. 7. Balance scores (expressed as TOC) during sustained wakefulness: subjects a-e. Mean linear regression of TOC against time awake $\left(F=26.5, P<0.01, R^{2}=0.59\right)$. Two hours of wakefulness corresponds to 08:00 in the sustained wakefulness condition. Error bars are $\pm 1 S D, C V$ is coefficient of variation $(S D /$ mean $\times 100 \%)$. The triangle indicates true time awake $( \pm 2.5 \mathrm{~h})$ at the time of the test, the dashed line shows the score level of the single test, whereas the box indicates time awake estimate. Time awakeestimation procedure: (1) plot reference curve - sustained wakefulness balance scores against known and increasing time awake, and against known local time, (2) superimpose current test score onto the reference curve, (3) locate candidate time awake estimates(s) -time awake-instant(s) where the test score equals the reference score(s), (4) accept the candidate time awake estimate whose local time is closest to that of the test recording. Example: in e, the test score intersects the reference curve (mean $\pm 1 S D$ ) at three time awakepoints: $6 \mathrm{~h}$ (at 12:00 hours local time), $10 \mathrm{~h}$ (at 16:00 hours local time), and $18 \mathrm{~h}$ (at 24:00 hours local time) generating three candidate test time awake estimates. The test was recorded at 17:30 local time, so the reference score at 16:00 hours is the closest local time candidate of the three, meaning that the time awake estimate is $10 \mathrm{~h}$. The estimate is successful as it is within $\pm 2.5 \mathrm{~h}$ of the true time awake. 


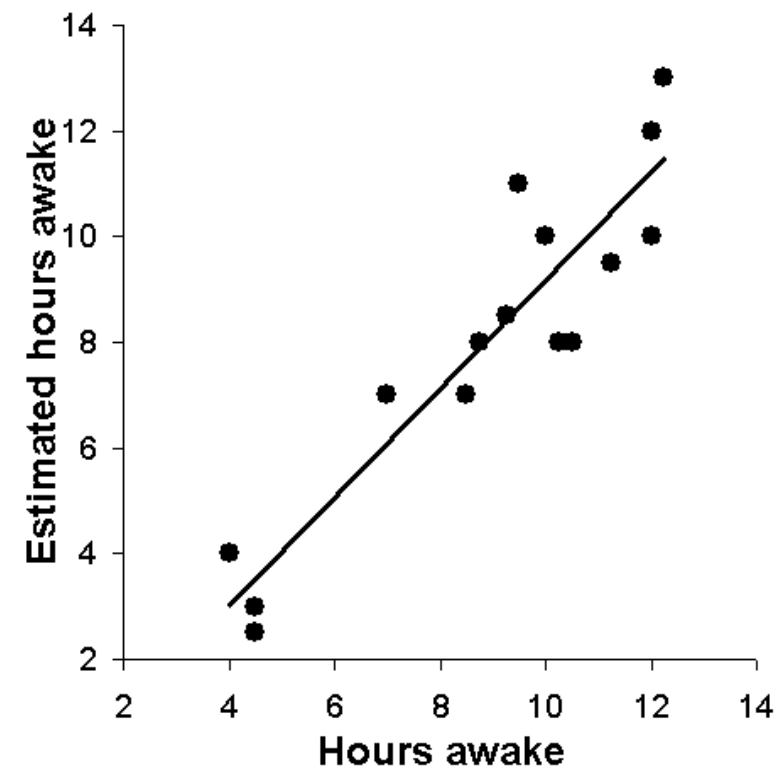

Fig. 8. Scatter plot and linear regression of estimated time awake against true time awake ( $F=74.2, P<0.0001, R^{2}=0.85, n=15$ subjects). This figure shows the correlation between real- and estimated time awake.

\subsection{Repeatability and Daytime Changes (Study III)}

The longitudinal set aimed at quantifying the test-retest reliability of the balance scores. The reliability was high, because the balance scores did not differ between weeks: $P \geq 0.05$ (Table 4).

The daytime set aimed at quantifying daytime dependent balance changes. From 8:30 to $13: 30$ hours, the changes were so significant, $P \leq 0.005$ (Fig. 9), that the scores did not overlap (Table 5).

This means that we reliably succeeded in quantifying the balance decline that precedes the general afternoon sleepiness peak.

Table 4. Balance scores during one month: once a week, same weekday, and at 8:30 hours -mean (SD), and ANOVA $P$-values $(n=30)$. This table shows the longitudinal repeatability of the balance scores.

\begin{tabular}{llllll}
\hline \hline Score & \multicolumn{4}{c}{ Mean (SD) } & \multicolumn{2}{c}{ ANOVA } \\
& Week 1 & Week 2 & Week 3 & Week 4 & $P$ \\
\hline $\begin{array}{l}F D \\
\left(\mathrm{~mm}^{2} / \mathrm{s}\right)\end{array}$ & $1.81(0.04)$ & $1.80(0.08)$ & $1.81(0.07)$ & $1.82(0.09)$ & 0.981 \\
$\begin{array}{l}M C A \\
(\mathrm{~mm})\end{array}$ & $10.7(0.8)$ & $10.8(0.7)$ & $10.9(0.6)$ & $10.7(0.7)$ & 0.967 \\
$\begin{array}{l}T O C \\
(\mathrm{~ms})\end{array}$ & $607(108)$ & $582(61)$ & $601(68)$ & $566(90)$ & 0.835 \\
\hline \hline
\end{tabular}


Table 5. Balance scores from 8:30 to 13:30 hours -mean $(S D)$ and linear regression of scores against time, $(n=30)$. This table shows the daytime changes of the balance scores.

\begin{tabular}{llllll}
\hline \hline Score & \multicolumn{3}{c}{ Mean (SD) } & \multicolumn{2}{c}{ Linear regression $^{\dagger}$} \\
& At 08:30 & At 10:30 & At 13:30 & Slope(CI) ${ }^{\dagger \dagger}$ Intercept $^{\ddagger}$ \\
\hline $\begin{array}{l}F D \\
\left(\mathrm{~mm}^{2} / \mathrm{s}\right)\end{array}$ & $1.87(0.03)$ & $1.81(0.06)$ & $1.75(0.07)$ & $-0.02(0.01)$ & 1.86 \\
$\begin{array}{l}M C A \\
(\mathrm{~mm})\end{array}$ & $10.5(0.4)$ & $11.2(0.4)$ & $11.8(0.2)$ & $0.25(0.05)$ & 10.6 \\
$\begin{array}{l}\text { TOC } \\
(\mathrm{ms})\end{array}$ & $587(33)$ & $550(23)$ & $487(41)$ & $-20.2(4.6)$ & 588 \\
\hline \hline
\end{tabular}

$n=90: 30$ subjects, 3 tests per subject

${ }^{\dagger \dagger}$ Regression line slopes $b$ : means and $95 \%$ CIs for 30 subjects. Slope unit: (parameter unit/hour). E.g. MCA increased $0.2-0.3 \mathrm{~cm}$ per hour. In $5 \mathrm{~h}$ it increased $1.25 \mathrm{~cm}(0.25 \mathrm{~cm} / \mathrm{h} \times 5 \mathrm{~h})$.

${ }^{*}$ Regression line intercepts $a$, parameter group mean values at 08.30.
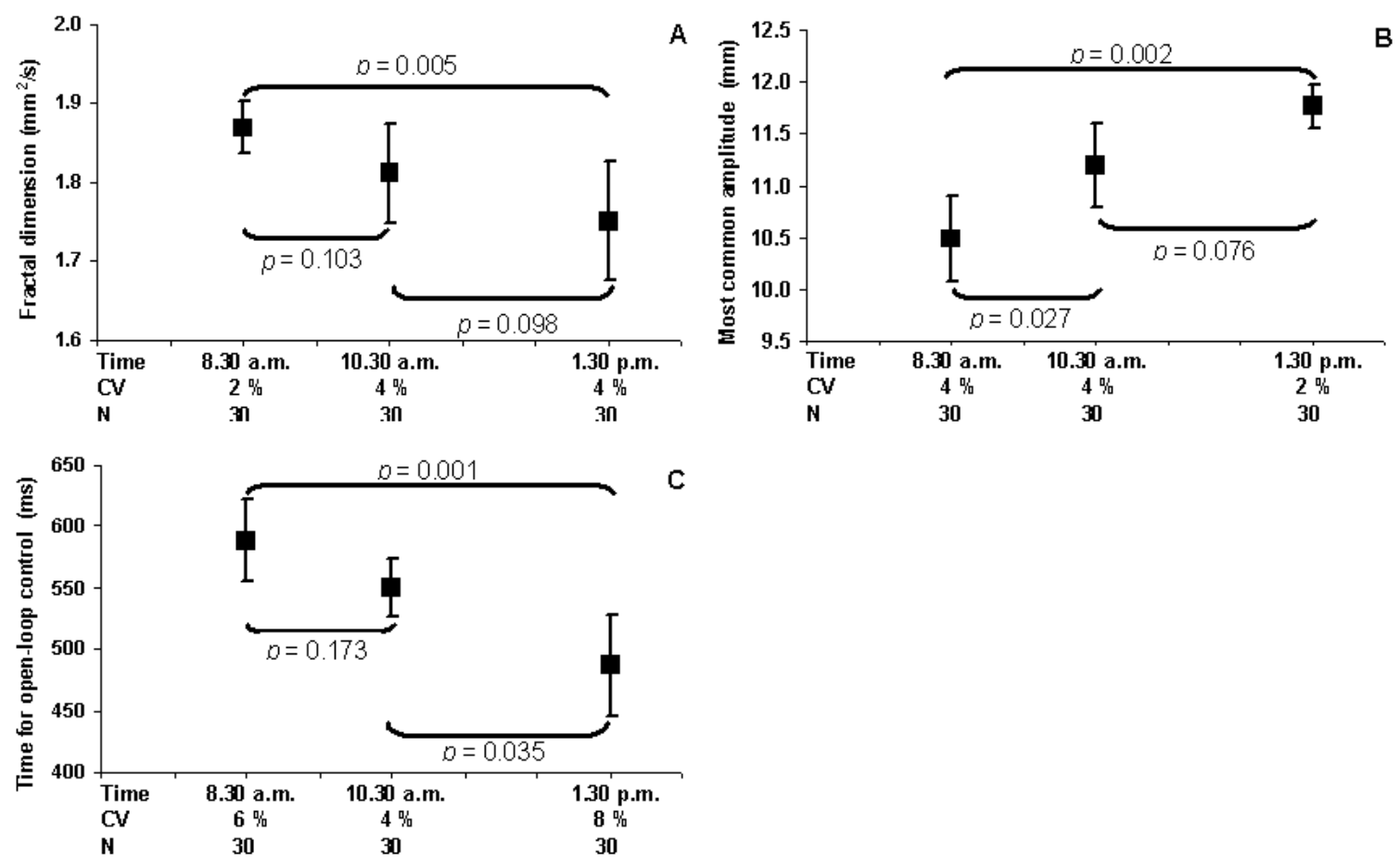

Fig. 9. Scatter plot and ANOVA of mean balance scores against local time for: a, $F D, \mathbf{b}, M C A$ and $\mathbf{c}, T O C$. $N=$ subjects; error bars are $\pm 1 S D ; C V$ is coefficient of variation $(S D /$ mean $\times 100 \%)$. This figure shows the statistical difference between balance scores recorded at different time of the day.

\subsection{Validity of Posturographic Time Awake Testing (Study IV)}

In the test set, 12 subjects were tested once a day for 7 days. Regression analysis showed significant $(P<0.0001)$ linear correlation between estimated time awake and true time awake, that roughly accounted for $70 \%$ of the variance (Fig. 10a). For each $1 \mathrm{~h}$ increase in 
true time awake, the estimated time awake increased by $0.88 \mathrm{~h}$ (i.e. $53 \mathrm{~min}$ ). The mean accuracy was $85.5 \pm 3.0 \%$ of the true time awake -corresponding to $96.5 \%$ precision. Regression analysis showed no correlation between days and accuracy (Fig. 11). The positive predictive value $(P P V)$ of the time awake estimate was $57.4 \%$, the negative predictive value (NPV) $91.4 \%$, sensitivity $55.6 \%$, and specificity $91.3 \%$.

In the longitudinal test set, 12 subjects were tested once a week for 4 weeks. Regression analysis showed a significant $(P<0.0001)$ linear correlation between the estimated time awake and the true time awake which roughly accounted for $70 \%$ of the variance (Fig. 10b). For each $1 \mathrm{~h}$ increase in true time awake, the estimated time awake increased by $0.87 \mathrm{~h}$ (i.e. $52 \mathrm{~min}$ ). The mean accuracy was $92.6 \pm 0.6 \%$ of the true time awake -corresponding to $99.3 \%$ precision. Regression analysis showed no correlation between weeks and accuracy (Fig. 11). The high accuracy means that the time awake tester estimates time awake accurately. The finding that the accuracy did not improve or impair with the testing days means that the precision (test-retest reliability) of the time awake tester is high. The PPV of the time awake estimate was $65.2 \%, N P V 97.6 \%$, sensitivity $82.9 \%$, specificity $93.9 \%$, and accuracy $91.7 \%$.
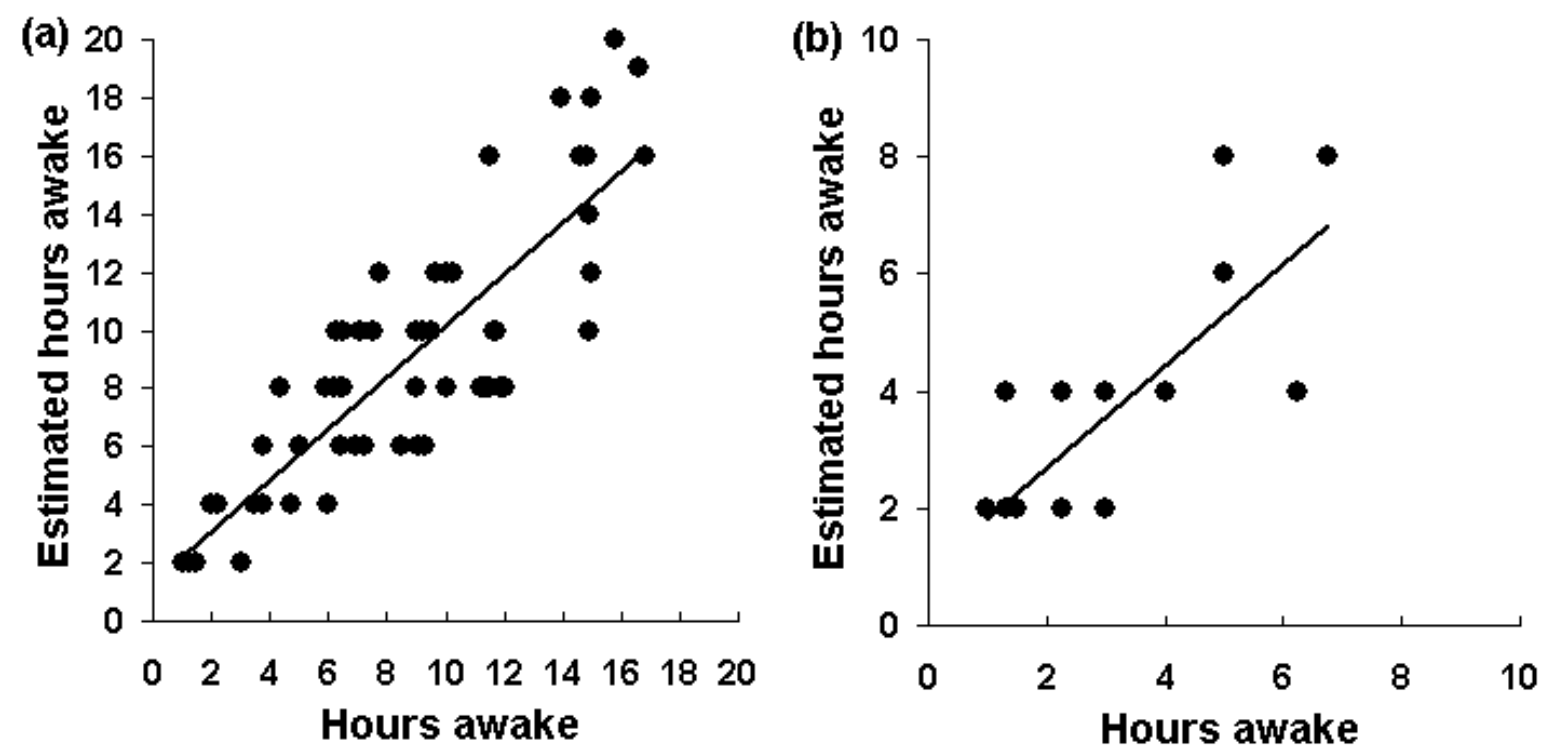

Fig. 10. Scatter plot and linear regression of estimated time awake against true time awake in: a) Test set data $\left(F=156, P<0.0001, R^{2}=0.73, n=84\right)$. The data was not heteroscedastic (F-test: $\left.F=0.94, P=0.41, n=84\right)$; and b) Longitudinal test set data $\left(F=72.1, P<0.0001, R^{2}=0.69, n=48\right)$. This figure shows the correlation between real- and estimated time awake. 


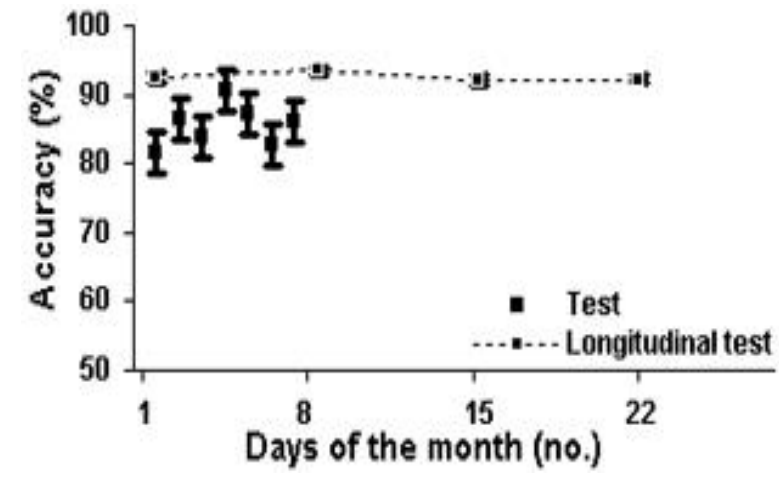

Fig. 11. Mean linear regression of time awake estimation accuracy against: days in the Test set (Mon to Sun, $F=0.36, P \geq 0.58, R^{2}=0.07, n=84$ ), and weeks in the Longitudinal test set (Weeks 1 to 4 , $\left.F=0.53, P \geq 0.54, R^{2}=0.21, n=48\right)$. Error bars $\pm 1 S D$. This figure shows the longitudinal repeatability of the test accuracy.

\subsection{Test Accuracy Versus Test Length (Study V)}

The aim was to quantify how the accuracy of a time awake test depends on the test length, and to identify the minimum required test length. Accuracy improved, - i.e. the $\Delta T$ values decreased- significantly with increasing test length (Fig. 12). Regression analysis showed that the correlation between accuracy and test length was significant, $P=0.0001$, and that the correlation accounted for $93 \%$ of the variance. For $2 \mathrm{~s}$ tests the accuracy was $12.7 \pm 4.5$ $\mathrm{h}$, for $j_{\min }=18 \mathrm{~s}$ tests the accuracy was $4.74 \pm 1.25 \mathrm{~h}$, and for $30 \mathrm{~s}$ tests it was $2.41 \pm 0.76 \mathrm{~h}$. Increasing the test length from $2 \mathrm{~s}$ to $18 \mathrm{~s}$ improved the mean accuracy by $0.47 \mathrm{~h} / \mathrm{s}$, whereas increasing the test length from $18 \mathrm{~s}$ to $30 \mathrm{~s}$ improved the mean accuracy by 0.18 $\mathrm{h} / \mathrm{s}$. The accuracies obtainable with $j_{\min }$ and $30 \mathrm{~s}$ tests differed significantly $(P=0.0004$; Student's t-test, 40 degrees of freedom; $t=4.23)$. This means that the test length of a posturographic time awake tester should be, at a minimum, $18 \mathrm{~s}$.

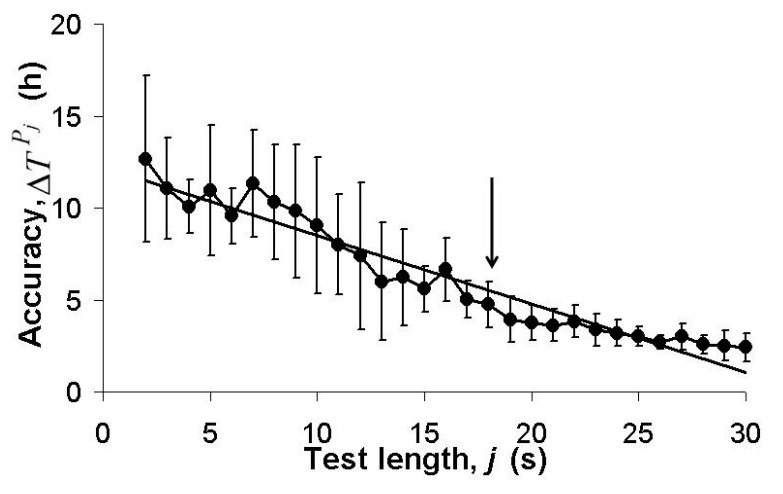

Fig. 12. Linear regression of accuracy against test length ( $F=368, P<0.0001, R^{2}=0.93,21$ subjects). The arrow indicates $j_{\min }$-the test length where the mean accuracy (eq. 4.3) went below $5 \mathrm{~h}$. The accuracy seemed to approach a plateau as the test length increased; variability across subjects seemed to decrease. Error bars $\pm 1 S D$. This figure shows the correlation between test sensitivity and test speed.

\subsection{Improved Test Validity (Study VI)}

In the test set, 12 subjects were tested once a day for 7 days. With the $C o M$ based balance scores, regression analysis showed significant $(P<0.0001)$ linear correlation between estimated time awake and true time awake, that roughly accounted for $80 \%$ of the variance (Fig. 13a). For each $1 \mathrm{~h}$ increase in true time awake, the estimated time awake increased by 
$0.93 \mathrm{~h}$ (i.e. $56 \mathrm{~min}$ ). The mean accuracy was $88.2 \pm 3.0 \%$ of the true time awake corresponding to $96.5 \%$ precision. Regression analysis showed no correlation between days and accuracy (Fig. 14). The PPV of the time awake estimate was $66.9 \%$, the NPV $92.2 \%$, sensitivity $59.4 \%$, and specificity $94.2 \%$.

In the longitudinal test set, 12 subjects were tested once a week for 4 weeks. Regression analysis showed a significant $(P<0.0001)$ linear correlation between the estimated time awake and the true time awake which roughly accounted for $70 \%$ of the variance (Fig. 13b). For each $1 \mathrm{~h}$ increase in true time awake, the estimated time awake increased by $0.72 \mathrm{~h}$ (i.e. $43 \mathrm{~min}$ ). The mean accuracy was $92.4 \pm 0.6 \%$ of the true time awake -corresponding to $97.2 \%$ precision. Regression analysis showed no correlation between weeks and accuracy (Fig. 14). The PPV of the time awake estimate was $92.2 \%$, NPV $92.3 \%$, sensitivity $60.7 \%$, and specificity $99.2 \%$.
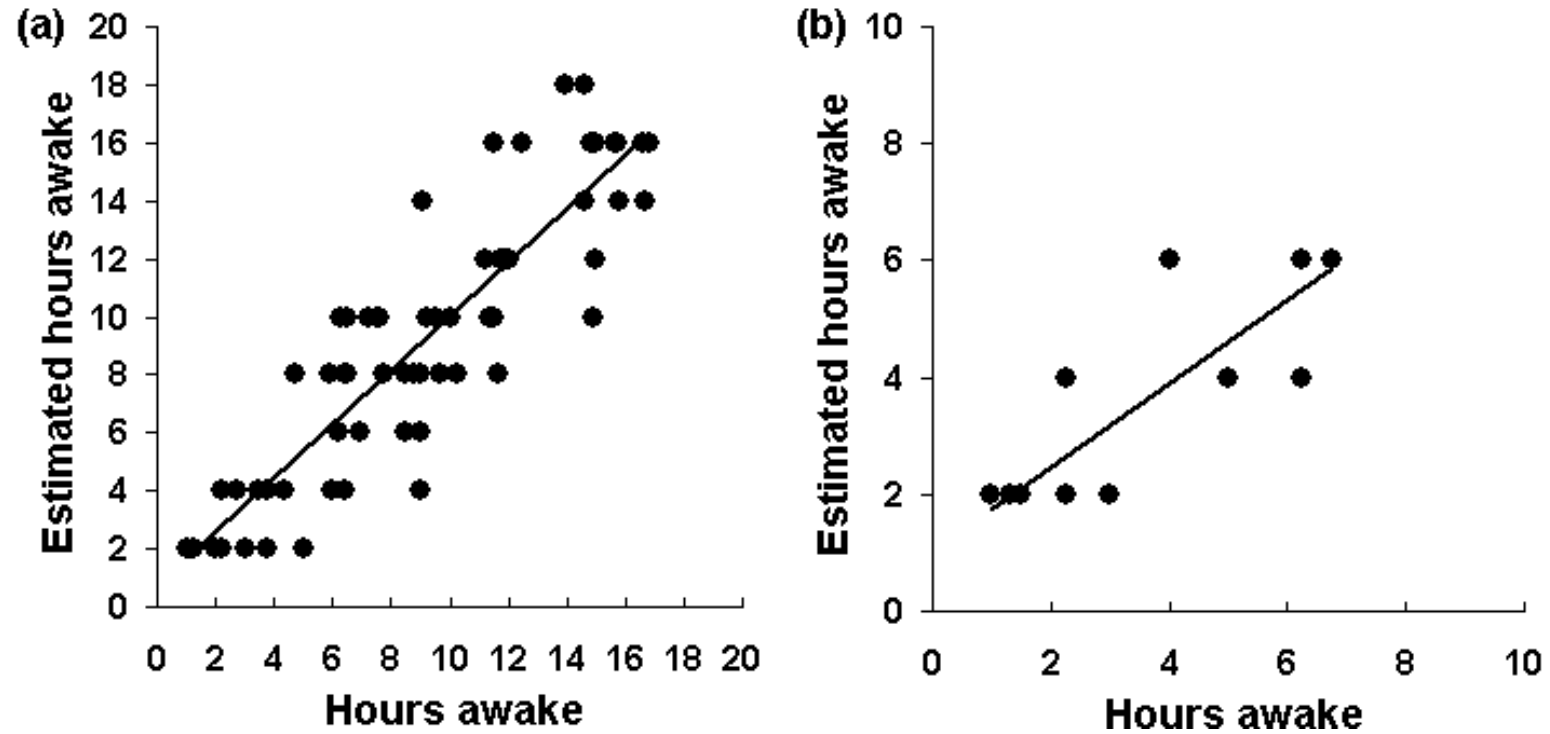

Fig. 13. Scatter plot and linear regression of estimated time awake against true time awake in: a) Test set data $\left(F=243, P<0.0001, R^{2}=0.80, n=84\right)$, and b) Longitudinal test set data $\left(F=90.8, P<0.0001, R^{2}=0.73\right.$, $n=48$ ). This figure shows the correlation between real- and estimated time awake.

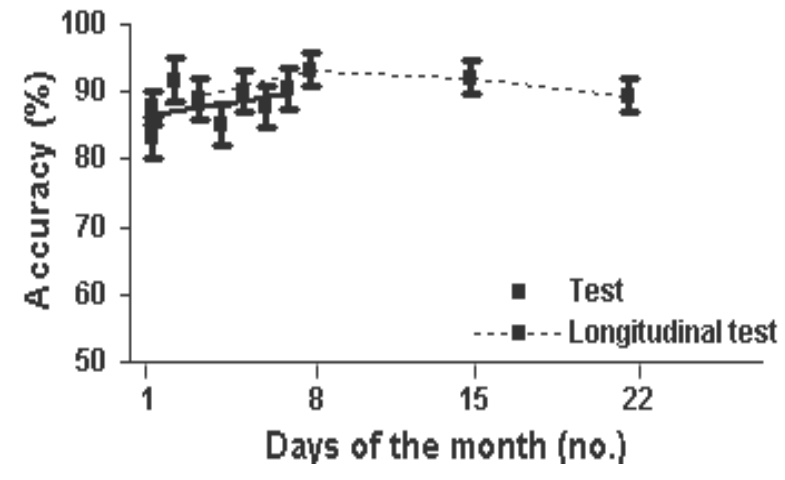

Fig. 14. Mean linear regression of time awake estimation accuracy against: days in the Test set (Mon to Sun, $F=0.86, P \geq 0.40, R^{2}=0.15, n=84$ ), and weeks in the Longitudinal test set (Weeks 1 to 4 , $\left.F=0.08, P \geq 0.80, R^{2}=0.04, n=48\right)$. Error bars $\pm 1 S D$. This figure shows the longitudinal repeatability of the test accuracy. 


\section{DISCUSSION}

\subsection{Need for Study and Contribution of the Thesis}

Sleepiness is frequently related to e.g. traffic- and occupational accidents [13-16, 28], because psychomotor performance declines during sustained wakefulness [1-3]. For instance, $17 \mathrm{~h}$ of wakefulness affects the performance as negatively as $0.5 \% \circ B A C$ does [13]. However, whereas breath-testers allow easy surveillance and facilitate legislative efforts against alcohol-related accidents, there is no simple sleepiness-tester [5-7]. Polls show that people drive while sleepy $[9,93]$, which implies that people are unaware of, or underrate the safety hazards of sleepiness. Hence, a sleepiness tester facilitates onsite safety monitoring as well as regulatory and legislative efforts to minimize the health and safety hazards of sleepiness $[6,8,22]$.

A test that predicts impending sleepiness levels would address safety issues related to sleepiness. An approach could be to measure time awake, because sleepiness increases as time awake increases $[23,25]$. Moreover, balance declines as time awake increases [8, 33, 46, 64-69]. These two relations advocate developing a posturographic time awake tester that could provide an estimate of sleepiness. Besides, the negative correlation between time awake and balance suggests posturography as a possible predictor of sleepiness related falls and slips, -an analogy to the promising use of posturography as a falls predictor among elderly [90].

This thesis examines posturographic time awake testing for instrumentation purposes. The motivation for this thesis is 1) that time awake is a relevant parameter in sleepiness and provides a sleepiness estimate, and 2) the hypothesis that time awake is posturographically estimable. The effect of sustained wakefulness on balance was identified and used to make time awake estimates.

\subsection{Quantifying Time Awake Posturographically}

Existing sleepiness tests quantify sleepiness by the magnitude of physiologic, manifest, or perceived sleepiness markers [5, 23, 33, 44-52]. Hence, the tests quantify sleepiness rather than time awake. The motive to quantify time awake is that sustained wakefulness increases the sleep homeostasis (sleep drive), which underlies sleepiness [23, 25]. Moreover, time awake is an easily understood variable: most people can record their wakeup time, and hence time awake provides a convenient and reliable proxy to validate the tester against. This also means that a time awake tester provides an everyman's tester -as compared to e.g. KSS or ESS that require skilled subjects to distinguish between sleepiness and other factors that affect performance [5]. Most important: a test that quantifies time awake will facilitate safety surveillance and regulations. Equating balance decline during sustained wakefulness with balance decline due to increasing $B A C$ provides authorities 
with two alternative posturographic thresholds: balance decline seen at a prohibited $B A C$, and balance decline seen at a length of sustained wakefulness that should be prohibited for safety reasons.

This thesis confirms that sustained wakefulness impairs balance (Studies I and III), as reported in previous studies on balance during sustained wakefulness [8, 33, 46, 64-69]. This thesis also shows that the posturographic test is valid with respect to the $C F F$ sleepiness test (Study I, Fig. 6). Figure 6 shows that both the balance and the $C F F$ exhibit a nadir at night, but that the balance nadir preceeds the $C F F$ nadir. The phase difference (ca. $4 \mathrm{~h}$ ) may imply -as the thesis assumed-that the tests address different sleepiness: the $C F F$ assesses manifest sleepiness whereas posturography is expected to assess physiologic sleepiness.

Valid time awake testing requires that the response to increasing time awake is monotone. This thesis support previous findings where increasing time awake explains $50 \%$ of the diurnal balance variations [65], but also shows that the balance parameters most sensitive to increasing time awake (Study I) explain even $60 \%$ of the diurnal balance variations (Studies II and IV). This fact allowed this thesis to show, that posturographic time awake estimates are accurate and precise -regardless whether one employs CoP based (Studies II-IV) or CoM based (Study VI) balance scores. The CoM based balance scores provide slightly improved accuracy of the time awake estimates compared to the CoP based balance scores.

The thesis showed that the balance scores $F D, M C A$, and TOC exhibited less dispersion across subjects (coefficient of variation, $\mathrm{CV}$, ranged $2 \%$ to $8 \%$, Fig. 9) than past studies on fatigue related balance changes have suggested (CV 13\% to 59\%) [8, 69]. Moreover, the individual test-retest variability was somewhat lower (CV $9 \%$ to $23 \%$ for 5 test repetitions, Fig. 7) than studies on test-retest variability suggest (CV $11 \%$ to $60 \%$ ) [94, 95]. The thesis also showed that the 'accuracy' (eq. 4.3) of posturographic time awake testing depends on test length (Study IV). Even an $18 \mathrm{~s}$ test identified time awake dependent balance changes. This supports a previous study where $15 \mathrm{~s}$ posturographic tests recorded diurnal balance variations [67]. However, a $40 \%$ speed-up (from 30 to $18 \mathrm{~s}$ ) of the test trades off accuracy, and may seem irrelevant when the duration of competing sleepiness tests range from 20 min for the MSLT [44, 45] to about 5 to $10 \mathrm{~min}$ for the PVT [50, 51].The motivation to examine posturography for fast, simple, and reliable time awake testing was that motivation and compensatory strategies bias balance less than existing sleepiness tests, and that the posturographic test can be designed not to involve task based performance. The high precision of the repeated time awake estimates in Study IV show that the personal factors did not influence the test. This is possible in quiet stance with feet together and arms crossed over the chest, because in this stance the CNS is likely to execute the balance process at an unconscious level [43]. 


\subsection{The Physiologic Link Between Balance and Successful Time Awake Testing}

The promising results of this thesis strongly suggest that posturography has potential for sleepiness testing. The technique is therefore worthy of further development efforts.

Eligible posturographic time awake testing requires that sleep homeostasis affects the physiology of balance [23]. Such physiologic impact then alters the mechanics of balance, alterations that we can measure. Hence, theoretically the answer lies in the biomechanics of balance, which can be modelled. First, one can model the body as an inverted pendulum (in this thesis a SLIPM, Fig. 4b). Second, one can add a control system to the model (e.g. the PID controller presented in Fig. 4b). Both approaches pool the functioning of the vestibular-, proprioceptic- and visual systems, the CNS control, and the muscular response into the posturographically measurable outcome, -the CoP. Dysfunction in any of these components due to sleepiness -separately or simultaneously- is possible. Moreover, control system models include time delays and noise sources into the process. The "noise" embodies factors that influence balance: e.g. circadian rhythm [46, 65], fatigue [8, 69], ageing [70-72], gender [72, 78], height [72, 78], balance disorders [73, 74], stimulants [33], alcohol, or drugs [79, 80]. When the consequences of dysfunction or time delay exceed the noise in the control process -with a sufficient $S N R$-the effects of the sleep homeostasis on balance can be quantified. Figure 7 shows that the TOC increases during sustained wakefulness. This implies that the balance control process reweighs towards open loop control -the time delay of the neural controller (Fig. 4b) increases. Such an increase worsens the balance as it allows more swaying. Interestingly, the same reweighing occurs in age-related balance changes [71]. This means that future research should compare age-related and sleepiness-related balance changes. Studies I-V did not presume a balance model -they examined the measured CoP trajectories. Study VI employed the passive SLIPM to remove the muscular response from the measured outcome. We applied the model to the $A P$ sway, but here we may have lost information, because $M L$ sway increases with the feet together [58], and $M L$ sway is a potential predictor of subsequent falls [90]. However, in quiet stance with the feet together the body mainly pivots around the ankle joint in the $A P$ direction -justifying the $A P$ choice. We also assumed that in quiet stance with the feet together, the body employs the ankle-strategy, although findings imply that the hip-strategy also is present [58]. Summing up, the SLIPM output rather represents CoM-related movements, but we showed that the SLIPM improved the correlation between TOC and time awake form $70 \%$ to $80 \%$. The improvement could partially stem from the fact that the range of motion for CoM is smaller than the range of motion for CoP. However, for the purpose of the time awake tester, the SLIPM is valid as a balance model.

\subsection{Limitations and Implications of the Thesis}

The limitations are as follows. First, the fact that the circadian rhythm explains even $40 \%$ of the diurnal balance changes (Studies II and IV) hampers estimating time awake. One 
issue is that since the circadian phase and amplitude are personal [29] (Fig. 7), each subject requires his/her own reference curve. Another issue is that to make accurate time awake estimates one must know and account for the time of day of the time awake test (Study II). Second, this thesis only focused on acute sleepiness. This limits the interpretation of the test applicability to cumulative sleepiness: ability to quantify such sleepiness is, however, important since severe cumulative sleepiness is common among e.g. exposed professions [96]. Future studies should develop the test for cumulative sleepiness testing, validate the test against the EEG measures of the MSLT test, and against the sleep quantity and quality with sleep diaries. The fact that the longitudinal balance scores were repeatable (Study III) means that the test-retest reliability dos not pose a problem when the test is employed to assess cumulative sleepiness. Third, in the tests sets (that estimated the subjects' time awake at the test) no true time awake exceeded $17 \mathrm{~h}$ (Study II and IV, Figs. 8 and 10). This limits the interpretation of the test accuracy beyond this amount of sustained wakefulness. This is, however, important since $17 \mathrm{~h}$ is the wakefulness threshold where the negative effects of sleepiness equal the negative effects of the legally proscribed $B A C$. Fourth, this thesis examined and validated test precision during one month. This limits the interpretation of the validity of the subjects' reference curves with respect to time (months, years); perhaps preserved estimation accuracy requires annual recalibration of the reference curve. Fifth, the subjects comprised healthy non-smokers, for whom we prohibited caffeine and alcohol intake. Hence, the results are unbiased by balance disorders, back-, leg-, and muscle disorders, stimulants, and toxins. Moreover, the results apply to subjects of similar age. Because all these factors bias posturographic test results, regulatory authorities must be aware of these particular limitations -i.e. divide test subjects into different groups- otherwise the effectiveness of the safety surveillance will be reduced. Sixth, we chose the objective $C F F$ test to assess the subjects' alertness because of its speed $(3 \mathrm{~min})$ as compared to the PVT (10 $\mathrm{min})$ [50, 51]. This limits the interpretation of the posturographic test validity, and further studies should validate the posturographic test results against standard sleepiness tests as the MSLT and PVT. Seventh, we did not employ objective or subjective tests -e.g. EEG and sleep diaries- to assess the subjects' quantity- and quality of sleep on the nights preceding the tests. This limits the interpretation of the level of cumulative sleepiness present at the study onset -and may be one reason why we found that each subject requires his/her own reference curve for time awake testing. To develop the test as a screening device for excessive sleepiness, further studies must validate the posturographic test against objective tests of cumulative sleepiness.

The findings of this thesis are relevant to regulatory and legislative authorities who aim to reduce the safety hazards of sleepiness. This thesis implies that posturographic time awake testing is valid at least for $17 \mathrm{~h}$ of wakefulness -the wakefulness threshold where the negative effects of sleepiness equal the negative effects of the legally proscribed $B A C$ [1]. The documented set-up and procedure allow study replicas in independent sleep labs - 
to confirm the test validity- and also allow implementing the test in occupational environments. Implementation is simple: the necessary equipment is available on the market, and quiet standing is testable on subjects of all ages. If acquiring personal reference tables is impossible, balance scores recorded during the general afternoon sleepiness peak between 13:00 and 14:00 hours will serve as a reference to detect excessive sleepiness (Study III). However, although the balance scores reliably quantify the decline in balance that precedes the general afternoon sleepiness peak, this concept relies on the assumption that the peak occurs at the same time for all subjects. Because the circadian phase differs between subjects [29], future studies should examine larger study populations and also validate the concept against reference tests as the MSLT and PVT. The finding that balance declines towards the afternoon sleepiness peak implies that posturography could apply as a screening test for sleep-related slips and falls.

\subsection{Future Research}

Further research needs to focus on study replicas with broader study populations and on including objective neurophysiologic measurements of alertness and sleepiness to address the validation issues concerning both acute- and cumulative sleepiness testing. Automating the analysis adds test credibility for end users but also needs validation. To achieve a statistical power of 1.00 in a study population identical to the one measured, at least 70 subjects are necessary (Study IV gives $P \leq 0.01, R^{2} \geq 0.50$, and 2 predictors). A replica should include about 200 subjects and three age groups. This validates the test against age. Selecting subjects from medical, aviation, and transportation occupations validates the test against professions where sleepiness testing is needed. An area of interest for food and pharmaceutical industries might be to validate the effect of stimulants and sedatives -the marketing of which is based on the energizing or sedating benefits. The current posturographic time awake tester is eligible for testing this aspect -the stimulating or sedating effect of e.g. caffeinated beverages, sleeping pills, anaesthetics, alcohol, etc.

To improve the time awake tester, further efforts should evaluate the feasibility of other generally employed balance parameters than those examined in this study. Improving the analysis technique further should also focus on modelling balance with control theory [86], because Study VI showed that the performance of the posturographic time awake tester improved when the muscular responses to CoM imbalances were removed from the measured balance functioning. Finally, there might be other physiologic markers that respond to sustained wakefulness -as e.g. salivary amylase seems to do [23].

\subsection{Conclusion}

Sleepiness is a major risk factor in e.g. transportation and occupational accidents -still, the lack of convenient sleepiness tests precludes testing impending sleepiness levels. This thesis showed that balance impairs during sustained wakefulness, and that the impairment 
is significant enough to allow estimating the time awake. The thesis developed an accurate, repeatable, and handy test that posturographically estimates the time awake of the test subject. One study showed the correlation between posturographic- and $C F F$ test results; thus the posturographic method is promising for further development as a screening test of excessive sleepiness. Testing time awake posturographically relies on personal reference curves, and on knowing and accounting for the time of day of the posturographic test. Testing for excessive sleepiness relies on comparing the test score with the threshold score at 13:30 hours. The results of the thesis yield a procedure that is important for authorities concerned with occupational safety. 


\section{REFERENCES}

1. Dawson, D. and Reid, K., Fatigue, alcohol and performance impairment. Nature, 1997. 388(6639): p. 235.

2. Lamond, N. and Dawson, D., Quantifying the performance impairment associated with fatigue. J Sleep Res, 1999. 8(4): p. 255-62.

3. Williamson, A. and Feyer, A., Moderate sleep deprivation produces impairments in cognitive and motor performance equivalent to legally prescribed levels of alcohol intoxication. Occup Environ Med, 2000. 57(10): p. 649-55.

4. Eberhart, R., Hu, X., and Foresman, B., Dangers of sleepiness and inattention while driving. J Am Osteopath Assoc, 2000. 100(8 Suppl): p. S9-14.

5. Guilleminault, C. and Brooks, S., Excessive daytime sleepiness: a challenge for the practising neurologist. Brain, 2001. 124(Pt 8): p. 1482-91.

6. Jones, C., Dorrian, J., and Rajaratnam, S., Fatigue and the criminal law. Ind Health, 2005. 43(1): p. 63-70.

7. Van Dongen, H., Vitellaro, K., and Dinges, D., Individual differences in adult human sleep and wakefulness: Leitmotif for a research agenda. Sleep, 2005. 28(4): p. 479-96.

8. Sato, O., et al., [Fatigue and stress of anesthesiologists at work--third report: investigation with a flicker photometer and a stabilo-meter]. Masui, 1995. 44(5): p. 674-8.

9. 2005 Sleep in America poll. 2005, National Sleep Foundation.

10. Workers on Flexible and Shift Schedules in 2004 Summary. 2005, U.S. Department of Labor, Bureau of Labor Statistics.

11. Parent-Thirion, A., et al., Fourth European Working Conditions Survey. 2007, European Foundation for the Improvement of Living and Working Conditions: Dublin.

12. Akerstedt, T., Work hours, sleepiness and the underlying mechanisms. J Sleep Res, 1995. 4(S2): p. 15-22.

13. Maycock, G., Sleepiness and driving: the experience of UK car drivers. J Sleep Res, 1996. 5(4): p. 229-37.

14. Horne, J. and Reyner, L., Sleep related vehicle accidents. BMJ, 1995. 310(6979): p. 565-7.

15. Dobbie, K., Fatigue related crashes: an analysis of fatigue related crashes on Australian roads using an operational definition of fatigue. 2002, Australian Transport Safety Bureau.

16. Leger, D., The Cost of Sleep-Related Accidents: A Report for the National Commission on Sleep Disorders Research. Sleep, 1994. 17(1): p. 84-93.

17. Bonnet, H. and Arand, D., We are Chronically Sleep Deprived. Sleep, 1995. 18(10): p. 908-911.

18. Fenton, R., Drowsy driving is big killer in U.S., in The Boston Globe. 2007: Boston.

19. Moran, T., Drowsy Driving Raises Legal Issues in The New York Times. 2007: New York.

20. Bruun, S., Sasi dömd till 70 dagsböter, in Hufvudstadsbladet. 2007: Helsinki.

21. Sudenniemi, S., Univaje tai uniapnea monen oudon kuolonkolarin taustalla, in Turun Sanomat. 2006: Turku.

22. Dawson, D. and McCulloch, K., Managing fatigue: it's about sleep. Sleep Med Rev, 2005. 9(5): p. 365-80. 
23. Seugnet, L., et al., Identification of a biomarker for sleep drive in flies and humans. Proc Natl Acad Sci U S A, 2006. 103(52): p. 19913-8.

24. Shen, J., Barbera, J., and Shapiro C., Distinguishing sleepiness and fatigue: focus on definition and measurement. Sleep Med Rev, 2006. 10(1): p. 63-76.

25. Borbely, A. and Achermann, P., Sleep homeostasis and models of sleep regulation. J Biol Rhythms, 1999. 14(6): p. 557-68.

26. Andreeva, E., et al., Driver Drowsiness Detection Using Multimodal Sensor Fusion. Proceedings of SPIE -- Multisensor, Multisource Information Fusion: Architectures, Algorithms, and Applications 2004, 2004. 5434: p. 380-390

27. Dement, W. and Carskadon, M., Current perspectives on daytime sleepiness: the issues. Sleep, 1982. 5 Suppl 2: p. S56-66.

28. Mitler, M., et al., Catastrophes, sleep, and public policy: consensus report. Sleep, 1988. 11(1): p. 100-9.

29. Baehr, E., Revelle, W., and Eastman, C., Individual differences in the phase and amplitude of the human circadian temperature rhythm: with an emphasis on morningness-eveningness. J Sleep Res, 2000. 9(2): p. 117-27.

30. Jewett, M., et al., Time course of sleep inertia dissipation in human performance and alertness. J Sleep Res, 1999. 8(1): p. 1-8.

31. Wertz, A., et al., Effects of sleep inertia on cognition. Jama, 2006. 295(2): p. 163-4.

32. Torsvall, L., et al., Sleep on the night shift: 24-hour EEG monitoring of spontaneous sleep/wake behavior. Psychophysiology, 1989. 26(3): p. 352-8.

33. Patat, A., et al., Effects of a new slow release formulation of caffeine on EEG, psychomotor and cognitive functions in sleep-deprived subjects. Hum Psychopharmacol, 2000. 15(3): p. 153-170.

34. Wilhelm, B., et al., Daytime variations in central nervous system activation measured by a pupillographic sleepiness test. J Sleep Res, 2001. 10(1): p. 1-7.

35. Richardson, G., et al., Circadian variation of sleep tendency in elderly and young adult subjects. Sleep, 1982. 5 Suppl 2: p. S82-94.

36. Mavjee, V. and Horne, J., Boredom effects on sleepiness/alertness in the early afternoon vs. early evening and interactions with warm ambient temperature. $\mathrm{Br} \mathrm{J}$ Psychol, 1994. 85 ( Pt 3): p. 317-33.

37. Roehrs, T., et al., Sedating Effects of Ethanol And Time of Drinking. Alcoholism: Clinical and Experimental Research 1992. 16(3): p. 553-557.

38. Wells, A., et al., Effects of meals on objective and subjective measures of daytime sleepiness. J Appl Physiol, 1998. 84(2): p. 507-15.

39. Westensen, N., W. Killigore, and T. Balkin, Performance and alertness effects of caffeine, dextroamphetamine, and modafinil during sleep deprivation. J Sleep Res, 2005. 14: p. 255-266.

40. Lovallo, W., et al., Caffeine Stimulation of Cortisol Secretion Across the Waking Hours in Relation to Caffeine Intake Levels. Psychosomatic Medicine, 2005. 67: p. 734-739.

41. Prosise, G., et al., Effects of abstinence from smoking on sleep and daytime sleepiness. Chest, 1994. 105(4): p. 1136-41.

42. Waller, D. and S. Levander, Smoking and Vigilance Psychopharmacology, 1980. 70(2): p. 131-136.

43. Mumenthaler, M. and Mattle, H., Neurology. 4 ed. 2004, Stuttgart: Georg Thieme Verlag. 
44. Carskadon, M., et al., Guidelines for the multiple sleep latency test (MSLT): a standard measure of sleepiness. Sleep, 1986. 9(4): p. 519-24.

45. Zwyghuizen-Doorenbos, A., et al., Test-retest reliability of the MSLT. Sleep, 1988. 11(6): p. 562-5.

46. Nakano, T., et al., Nineteen-hour variation of postural sway, alertness and rectal temperature during sleep deprivation. Psychiatry Clin Neurosci, 2001. 55(3): p. 277-8.

47. Wright, K., Hull, J., and Czeisler, C., Relationship between alertness, performance, and body temperature in humans. Am J Physiol Regul Integr Comp Physiol, 2002. 283: p. R1370-R1377.

48. Leproult, R., et al., Sleep loss results in an elevation of cortisol levels the next evening. Sleep, 1997. 20(10): p. 865-70.

49. Shreter, R., Peled, R., and Pillar, G., The 20-min trial of the maintenance of wakefulness test is profoundly affected by motivation. Sleep Breath, 2006. 10(4): p. 173-9.

50. Sallinen, M., et al., The effects of sleep debt and monotonous work on sleepiness and performance during a 12-h dayshift. J Sleep Res, 2004. 13(4): p. 285-94.

51. Urrila, A., et al., Psychomotor vigilance task performance during total sleep deprivation in young and postmenopausal women. Behavioural Brain Research, 2007. 180(1): p. 42-47.

52. Kaida, K., et al., Validation of the Karolinska sleepiness scale against performance and EEG variables. Clin Neurophysiol, 2006. 117(7): p. 1574-81.

53. Winter, D., et al., Stiffness control of balance in quiet standing. J Neurophysiol, 1998. 80(3): p. 1211-21.

54. Nashner, L., Strategies for Organization of Human Posture, in Vestibular and Visual Control on Posture and Locomotor Equilibrium, M. Igarashi and F. Black, Editors. 1985, Karger: Basel.

55. Winter, D., Biomechanics and Motor Control of Human Movement. 2004: John Wiley \& Sons.

56. van der Linden, M., et al., Muscle Reflexes and Synergies Triggered by an Unexpected Support Surface Height During Walking. J Neurophysiol, 2007. 97: p. 3639-3650.

57. Horak, F. and Nashner, L., Central programming of postural movements: adaptation to altered support-surface configurations. J Neurophysiol, 1986. 55(6): p. $1369-81$.

58. Day, B., et al., Effect of vision and stance width on human body motion when standing: implications for afferent control of lateral sway. J Physiol, 1993. 469: p. 479-99.

59. Barin, K., Evaluation of a generalized model of human postural dynamics and control in the sagittal plane. Biol Cybern, 1989. 61(1): p. 37-50.

60. Baloh, R. and Honrubia, V., Clinical Neurophysiology of the Vestibular System 2001: Oxford University Press.

61. Tsang, W. and Hui-Chan, C., Effects of exercise on joint sense and balance in elderly men: Tai Chi versus golf. Med Sci Sports Exerc, 2004. 36(4): p. 658-67.

62. Wade, M. and Jones, G., The role of vision and spatial orientation in the maintenance of posture. Phys Ther, 1997. 77(6): p. 619-28.

63. Redfern, M., Yardley, L., and Bronstein, A., Visual influences on balance. J Anxiety Disord, 2001. 15(1-2): p. 81-94. 
64. Morad, Y., et al., Posturography as an indicator of fatigue due to sleep deprivation. Aviat Space Environ Med, 2007. 78(9): p. 859-63.

65. Avni, N., et al., Brief posturographic test as an indicator of fatigue. Psychiatry Clin Neurosci, 2006. 60(3): p. 340-6.

66. Liu, Y., Higuchi, S., and Motohashi, Y., Changes in postural sway during a period of sustained wakefulness in male adults. Occup Med (Lond), 2001. 51(8): p. 490-5.

67. Gribble, P. and Hertel, J., Changes in postural control during a 48-hr. sleep deprivation period. Percept Mot Skills, 2004. 99(3 Pt 1): p. 1035-45.

68. Patel, M., et al., Effects of 24- $h$ and 36-h sleep deprivation on human postural control and adaptation. Exp Brain Res, 2008. 185(2): p. 165-73.

69. Kohen-Raz, R., et al., An initial evaluation of work fatigue and circadian changes as assessed by multiplate posturography. Percept Mot Skills, 1996. 82(2): p. 54757.

70. Prieto, T., Myklebust, J., and Myklebust, B., Characterization and Modeling of Postural Steadiness in the Elderly: A Review. IEEE Trans Rehabil Eng, 1993. 1(1).

71. Collins, J., et al., Age-related changes in open-loop and closed-loop postural control mechanisms. Exp Brain Res, 1995. 104(3): p. 480-92.

72. Era, P., et al., Postural balance in a random sample of 7,979 subjects aged 30 years and over. Gerontology, 2006. 52(4): p. 204-13.

73. Tossavainen, T., et al., Virtual reality in posturography. IEEE Trans Inf Technol Biomed, 2006. 10(2): p. 282-92.

74. Jacobs, J., et al., Multiple balance tests improve the assessment of postural stability in subjects with Parkinson's disease. J Neurol Neurosurg Psychiatry, 2006. 77(3): p. 322-6.

75. Gerbino, P., Griffin, E., and Zurakowski, D., Comparison of standing balance between female collegiate dancers and soccer players. Gait Posture, 2007. 26(4): p. 501-7.

76. Era, P., et al., Postural stability and skilled performance--a study on top-level and naive rifle shooters. J Biomech, 1996. 29(3): p. 301-6.

77. Nardone, A., et al., Fatigue effects on body balance. Electroencephalogr Clin Neurophysiol, 1997. 105(4): p. 309-20.

78. Chiari, L., Rocchi, L., and Cappello, A., Stabilometric parameters are affected by anthropometry and foot placement. Clin Biomech (Bristol, Avon), 2002. 17(9-10): p. 666-77.

79. Liguori, A., et al., Alcohol Effects on Mood, Equilibrium, and Simulated Driving. Alcoholism: Clinical and Experimental Research, 1999. 23(5): p. 815-821.

80. Ahmad, S., et al., Effects of lifetime ethanol consumption on postural control: a computerized dynamic posturography study. J Vestib Res, 2002. 12(1): p. 53-64.

81. Kerrigan, S. and Lindsey, T., Fatal caffeine overdose: Two case reports. Forensic Science International, 2005. 153(1): p. 67-69.

82. Kapteyn, T., et al., Standardization in platform stabilometry being a part of posturography. Agressologie, 1983. 24(7): p. 321-6.

83. Baratto, L., et al., A new look at posturographic analysis in the clinical context: sway-density versus other parameterization techniques. Motor Control, 2002. 6(3): p. 246-70.

84. Rocchi, L., Chiari, L., and Cappello, A., Feature selection of stabilometric parameters based on principal component analysis. Med Biol Eng Comput, 2004. 42(1): p. 71-9. 
85. Prieto, T., et al., Measures of postural steadiness: differences between healthy young and elderly adults. IEEE Trans Biomed Eng, 1996. 43(9): p. 956-66.

86. Peterka, R., Postural control model interpretation of stabilogram diffusion analysis. Biol Cybern, 2000. 82(4): p. 335-43.

87. Ishizaki, H., et al., Repeatability and effect of instruction of body sway. Acta Otolaryngol Suppl, 1991. 481: p. 589-92.

88. Uimonen, S., et al., The repeatability of posturographic measurements and the effects of sleep deprivation. J Vestib Res, 1994. 4(1): p. 29-36.

89. Goldie, P., Bach, T., and Evans, O., Force platform measures for evaluating postural control: reliability and validity. Arch Phys Med Rehabil, 1989. 70: p. 510517.

90. Piirtola, M. and Era, P., Force platform measurements as predictors of falls among older people - a review. Gerontology, 2006. 52(1): p. 1-16.

91. Toppila, E., et al., Effect of styrene on postural stability among reinforced plastic boat plant workers in Finland. J Occup Environ Med, 2006. 48(2): p. 175-80.

92. Wonnacott, R. and Wonnacott, T., Introductory Statistics, 4th Edition. 1985, New York: JOHN WILEY \& SONS.

93. Oron-Gilad, T. and Shinar, D., Driver fatigue among military truck drivers. Transportation Research Part F: Traffic Psychology and Behaviour, 2000. 3(4): p. 195-209.

94. Thyssen, H., et al., Normal ranges and reproducibility for the quantitative Romberg's test. Acta Neurol Scand, 1982. 66(1): p. 100-4.

95. Raymakers, J., Samson, M., and Verhaar H., The assessment of body sway and the choice of the stability parameter(s). Gait \& Posture, 2005. 21(1): p. 48-58.

96. Hanowski, R., et al., The sleep of commercial vehicle drivers under the 2003 hoursof-service regulations. Accid Anal Prev, 2007. 39(6): p. 1140-5. 
